\title{
NEAR SHARP STRICHARTZ ESTIMATES WITH LOSS IN THE PRESENCE OF DEGENERATE HYPERBOLIC TRAPPING
}

\author{
HANS CHRISTIANSON
}

\begin{abstract}
We consider an $n$-dimensional spherically symmetric, asymptotically Euclidean manifold with two ends and a codimension 1 trapped set which is degenerately hyperbolic. By separating variables and constructing a semiclassical parametrix for a time scale polynomially beyond Ehrenfest time, we show that solutions to the linear Schrödiner equation with initial conditions localized on a spherical harmonic satisfy Strichartz estimates with a loss depending only on the dimension $n$ and independent of the degeneracy. The Strichartz estimates are sharp up to an arbitrary $\beta>0$ loss. This is in contrast to CW11, where it is shown that solutions satisfy a sharp local smoothing estimate with loss depending only on the degeneracy of the trapped set, independent of the dimension.
\end{abstract}

\section{INTRODUCTION}

It is well known that there is an intricate interplay between the existence of trapped geodesics, those which do not escape to spatial infinity, and dispersive estimates for the associated quantum evolution. Trapping can occur in many different ways, from a single trapped geodesic (see [Bur04, BZ04, Chr07, Chr11, Chr08b]), to a thin fractal trapped set (see [NZ09, Chr09, Chr08a, Dat09]), to codimension 1 trapped sets in general relativity (see, for example, BS03, BH08, DR09, MMTT10, Luk10, TT11, LMar,WZ10, and the references therein), to elliptic trapped sets and boundary value problems. Dispersive type estimates also come in many flavors, but are all designed to express in some manner that the mass of a wave function tends to spread out as the wave function evolves. Since the mass of wave functions tends to move along the geodesic flow, the presence of trapped geodesics suggests some residual mass may not spread out, or may spread out more slowly than in the non-trapping case. In this paper, we concentrate on Strichartz estimates, and exhibit a class of manifolds for which we prove near-sharp Strichartz estimates with a loss depending only on the dimension of the trapped set. This class of manifolds has already been studied in CW11, where a sharp local smoothing estimate is obtained with a loss depending only on how flat the manifold is near the trapped set. This presents an interesting dichotomy conjecture: "loss in local smoothing depends only on the kind of trapping, while loss in Strichartz estimates depends only on the dimension of trapping".

The purpose of this paper is to study a very simple class of manifolds with a hypersphere of trapped geodesics. If the dynamics near such a sphere are strictly hyperbolic in the normal direction, then resolvent estimates are already obtained in [WZ10] (see also Chr08b, Chr08a]) which can be used to prove local smoothing estimates with only a logarithmic loss. However, if the dynamics are only weakly hyperbolic, resolvent estimates and local smoothing estimates are obtained 
in CW11 with a sharp polynomial loss in both. We now turn our attention to studying Strichartz estimates, which are mixed $L^{p} L^{q}$ time-space estimates. The typical procedure for proving Strichartz estimates is to construct parametrices (approximate solutions), which encode how wave packets move with the geodesic flow. For solutions of the Schrödinger equation, wave packets at higher frequency move at a higher velocity, so the presence of trapping, or more precisely of conjugate points means that parametrices can typically be constructed only for time intervals depending on the frequency of the wave packet. Then summing up many parametrices to get an estimate on a fixed time scale leads to derivative loss in Strichartz estimates.

However, if the trapped set is sufficiently thin and hyperbolic, we expect that most of a wave packet still propagates away quickly, and a procedure developed by Anantharaman Ana08 allows one to exploit this to logarithmically extend the timescale on which one can construct a parametrix leading to Strichartz estimates with no loss BGH10.

For the manifolds studied in this paper, the trapping is degenerately hyperbolic, so we still expect some mass of each wave packet to propagate away, but at a much slower rate than the strictly hyperbolic case. As a consequence, we need to extend the parametrix polynomially in time to get sharp estimates. The techniques in Ana08, BGH10, will not work in this situation since the $\mathcal{O}\left(h^{\infty}\right)$ estimate of decaying correlations will not control the exponential number of such correlations. In this paper, we fail to prove estimates all the way to the sharp polynomial timescale, but we are nevertheless able to extend the parametrix construction to the sharp timescale up to an arbitrary $\beta>0$ loss, which is expressed as a loss in derivative in the main theorem. Further, the technique of proof involves decomposing the solution in terms of spherical harmonics in order to reduce the problem to a 1-dimensional semiclassical parametrix construction. Lacking a square-function estimate for spherical harmonics, the proof only works for initial data localized along one spherical harmonic eigenspace. In this sense, the result shows more about the natural semiclassical timescale, polynomially extended beyond Ehrenfest time, for which we have good control of the rate of dispersion.

We begin by describing the geometry. We consider $X=\mathbb{R}_{x} \times \mathbb{S}_{\theta}^{n-1}$, equipped with the metric

$$
g=d x^{2}+A^{2}(x) G_{\theta},
$$

where $A \in \mathcal{C}^{\infty}$ is a smooth function, $A \geqslant \epsilon>0$ for some epsilon, and $G_{\theta}$ is the metric on $\mathbb{S}^{n-1}$. From this metric, we get the volume form

$$
d \mathrm{Vol}=A(x)^{n-1} d x d \sigma,
$$

where $\sigma$ is the usual surface measure on the sphere. The Laplace-Beltrami operator acting on 0 -forms is computed:

$$
\Delta f=\left(\partial_{x}^{2}+A^{-2} \Delta_{\mathbb{S}^{n-1}}+(n-1) A^{-1} A^{\prime} \partial_{x}\right) f,
$$

where $\Delta_{\mathbb{S}^{n-1}}$ is the (non-positive) Laplace-Beltrami operator on the sphere.

We study the case $A(x)=\left(1+x^{2 m}\right)^{1 / 2 m}, m \geqslant 2$, in which case the manifold is asymptotically Euclidean (with two ends), and has a trapped hypersphere at the unique critical point $x=0$. Since $A(x)$ has a degenerate minimum at $x=0$, the trapped sphere is weakly normally hyperbolic in the sense that it is unstable and isolated, but degenerate (see Figure 1). 
Our main theorem is the following, which expresses that a solution of the linear homogeneous Schrödinger equation on this manifold satisfies Strichartz estimates with loss depending only on the dimension $n$, up to an arbitrary $\beta>0$ loss.

Theorem 1. Suppose u solves

$$
\left\{\begin{array}{l}
\left(D_{t}+\Delta\right) u=0 \\
\left.u\right|_{t=0}=u_{0}
\end{array}\right.
$$

where $u_{0}=H_{k} u_{0}$ is localized on the spherical harmonic subspace of order $k$. Then for any $T, \beta>0$, there exists $C_{T, \beta}>0$ such that

$$
\|u\|_{L^{p}([0, T]) L^{q}(M)} \leqslant C_{T, \beta}\left\|\left\langle D_{\theta}\right\rangle^{(n-2) / p n+\beta} u_{0}\right\|_{L^{2}(M)},
$$

where

and $2 \leqslant q<\infty$ if $n=2$.

$$
\frac{2}{p}+\frac{n}{q}=\frac{n}{2}
$$

Remark 1.1. There are several important observations to make about Theorem 1. First, this theorem concerns endpoint Strichartz estimates. In dimension $n \geqslant 3$, if we take $\beta<1 / n$, the loss in derivative is then $(n-2) / 2 n+\beta<1 / 2$; that is, the loss is always less than the loss following the argument of Burq-GérardTzvetkov BGT04 (which gives a loss of $1 / 2$ for endpoint estimates in $n \geqslant 3$ ). Second, there is only a $\beta>0$ loss over the Euclidean (scale-invariant) estimates if $n=2$, that is, if the trapped set is a single degenerate periodic geodesic, so we can get as close to the no-loss estimates as we like. We expect the $\beta>0$ derivative loss can actually be removed in all dimensions, but this is beyond our techniques. Third, in all dimensions, the loss depends only on the dimension of the trapped set. It does not depend on $m$, the order of degeneracy of the trapping. This is in sharp contrast to the local smoothing effect, which depends only on $m$, and not on the dimension $n$ (see CW11 and below).

For dimensions $n \geqslant 3$, the estimate (1.2) is near sharp on natural semiclassical time scales (see Corollary [3.3), in the sense that no better polynomial derivative estimate can hold. In dimension $n=2$, the same is true by comparing to the scale-invariant case.

Finally, since $u_{0}$ is localized to a single spherical harmonic, the estimate in the theorem can be rephrased, since

$$
\left\|\left\langle D_{\theta}\right\rangle^{(n-2) / p n+\beta} u_{0}\right\|_{L^{2}(M)} \sim\left\|\langle k\rangle^{(n-2) / p n+\beta} u_{0}\right\|_{L^{2}(M)} .
$$

Acknowledgements. The author would like to thank Nicolas Burq, Kiril Datchev, Colin Guillarmou, Jeremy Marzuola, Jason Metcalfe, Fabrice Planchon, and Michael Taylor for helpful and stimulating conversations during the preparation of this manuscript.

\section{Reduction In Dimension}

In this section we use a series of known techniques and estimates to reduce study of the Schrödiner equation on $M$ to the study of a semiclassical Schrödiner equation on $\mathbb{R}$ with potential. The potential has a degenerate critical point, and we use a technical blow-up calculus to construct a sequence of parametrices near the critical point. 


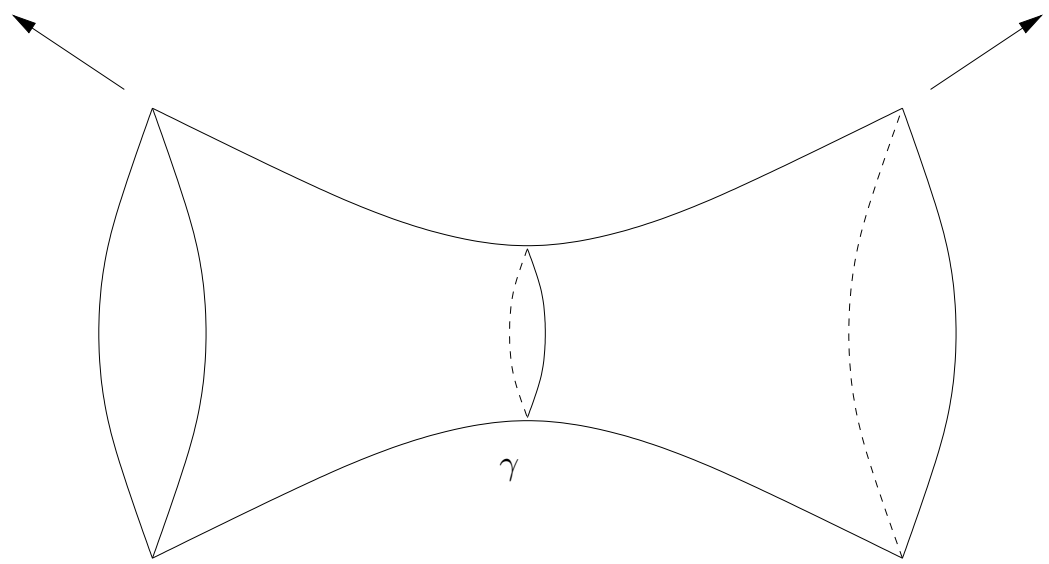

Figure 1. A piece of the manifold $X$ and the trapped sphere at $x=0$.

We observe that we can conjugate $\Delta$ by an isometry of metric spaces and separate variables so that spectral analysis of $\Delta$ is equivalent to a one-variable semiclassical problem with potential. That is, let $T: L^{2}(X, d \mathrm{Vol}) \rightarrow L^{2}(X, d x d \theta)$ be the isometry given by

$$
T u(x, \theta)=A^{(n-1) / 2}(x) u(x, \theta) .
$$

Then $\widetilde{\Delta}=T \Delta T^{-1}$ is essentially self-adjoint on $L^{2}(X, d x d \sigma)$ for our choice of $A$. A simple calculation gives

$$
-\widetilde{\Delta} f=\left(-\partial_{x}^{2}-A^{-2}(x) \Delta_{\mathbb{S}^{n-1}}+V_{1}(x)\right) f,
$$

where the potential

$$
V_{1}(x)=\frac{n-1}{2} A^{\prime \prime} A^{-1}+\frac{(n-1)(n-3)}{4}\left(A^{\prime}\right)^{2} A^{-2} .
$$

Of course, conjugating the Laplacian by an $L^{2}$ isometry does not necessarily preserve $H^{s}$ or $L^{q}$ spaces.

Lemma 2.1. With the notation $A(x)=\left(1+x^{2 m}\right)^{1 / 2 m}$ from above, for $s \geqslant 0$,

and for $q \geqslant 2$,

$$
\begin{gathered}
\|T u\|_{H^{s}(d x d \sigma)} \leqslant C\|u\|_{H^{s}(d V o l)}, \\
\left\|\left\langle-\Delta_{\mathbb{S}^{n-1}}\right\rangle^{s} T u\right\|_{L^{2}(d x d \sigma)}=\left\|\left\langle-\Delta_{\mathbb{S}^{n-1}}\right\rangle^{s} u\right\|_{L^{2}(d V o l)},
\end{gathered}
$$

$$
\|u\|_{L^{q}(d V o l)} \leqslant C\|T u\|_{L^{q}(d x d \sigma)} .
$$

Proof. The result for $0<s \leqslant 1$ follows from the $L^{2}$ and $H^{1}$ result, which follows by observing that

$$
\partial_{x} A^{(n-1) / 2} u=A^{(n-1) / 2} \partial_{x} u+\frac{(n-1)}{2} A^{\prime} A^{(n-3) / 2} u .
$$

But since $\left|A^{\prime}\right| \leqslant C A$, the $L^{2}(d x d \sigma)$ norm of $\partial_{x} T u$ is bounded by the $H^{1}(d \mathrm{Vol})$ norm of $u$.

The result for angular derivatives follows by commuting with $A^{(n-1) / 2}(x)$. 
For the $L^{q}$ result, we compute

$$
\int|u(x, \theta)|^{q} A^{(n-1)}(x) d x d \sigma=\int\left|A^{(n-1)(1 / q-1 / 2)} T u(x, \theta)\right|^{q} d x d \theta .
$$

The function $A^{(n-1)(1 / q-1 / 2)}(x)$ is bounded for $q \geqslant 2$, so the $L^{q}$ inequality is true as well.

As a consequence of this, to prove Theorem [1, it suffices to prove the following Proposition, and apply Lemma 2.1 with $v=T u$.

Proposition 2.2. Suppose $v$ solves

$$
\left\{\begin{array}{l}
\left(D_{t}+\widetilde{\Delta}\right) v=0, \\
\left.v\right|_{t=0}=v_{0},
\end{array}\right.
$$

where $v_{0}=H_{k} v_{0}$ is localized on the spherical harmonic subspace of order $k$. Then for any $T>0$ and $\beta>0$, there exists $C_{T, \beta}>0$ such that

$$
\|v\|_{L^{p}([0, T]) L^{q}} \leqslant C_{T, \beta}\left\|\left\langle D_{\theta}\right\rangle^{(n-2) / p n+\beta} v_{0}\right\|_{L^{2}}
$$

where

and $2 \leqslant q<\infty$ if $n=2$.

$$
\frac{2}{p}+\frac{n}{q}=\frac{n}{2}
$$

We now separate variables by projecting onto the $k$ th spherical harmonic eigenspace. That is, let $\mathcal{H}_{k}$ be the $k$ th eigenspace of spherical harmonics, so that $v \in \mathcal{H}_{k}$ implies

$$
-\Delta_{\mathbb{S} n-1} v=\lambda_{k}^{2} v
$$

where

$$
\lambda_{k}^{2}=k(k+n-2)
$$

Let $H_{k}: L^{2}\left(\mathbb{S}^{n-1}\right) \rightarrow \mathcal{H}_{k}$ be the projector.

Since $v_{0}$ is assumed to satisfy $v_{0}=H_{k} v_{0}$ for some $k$ and the conjugated Laplacian preserves spherical harmonic eigenspaces, we have also $v=H_{k} v$. Motivated by spectral theory, we compute:

$$
\left(-\widetilde{\Delta}-\lambda^{2}\right) v=P_{k} v
$$

where

$$
P_{k} v=P_{k} H_{k} v=\left(-\frac{\partial^{2}}{\partial x^{2}}+k(k+n-2) A^{-2}(x)+V_{1}(x)-\lambda^{2}\right) v .
$$

Setting $h=(k(k+n-2))^{-1 / 2}$ and rescaling, we have the one-dimensional semiclassical operator

$$
P(z, h) \psi(x)=\left(-h^{2} \frac{d^{2}}{d x^{2}}+V(x)-z\right) \psi(x),
$$

where the potential is

$$
V(x)=A^{-2}(x)+h^{2} V_{1}(x)
$$

and the spectral parameter is $z=h^{2} \lambda^{2}$.

For our case where $A(x)=\left(1+x^{2 m}\right)^{1 / 2 m}$, the subpotential $h^{2} V_{1}$ is seen to be lower order in both the semiclassical and scattering sense. Furthermore, the principal potential $A^{-2}(x)$ is even, smooth, decays like $x^{-2}$ at $\pm \infty$ and has a unique degenerate maximum of the form $1-x^{2 m}$ at $x=0$. 


\section{Endpoint Strichartz estimates}

Before proceeding to the endpoint Strichartz estimates, let us briefly recall the local smoothing estimates which will eventually allow us to glue together Strichartz estimates on semiclassical timescales.

3.1. Local smoothing estimates. In this subsection, we recall the local smoothing estimates from [CW11, as well as the dual versions which we will use in this paper.

Theorem 2 ( CW11). Let $V(x)=A^{-2}(x)+h^{2} V_{1}(x)$ as above. Then for any $T>0$, there exists a constant $C=C_{T}>0$ such that

$$
\int_{0}^{T}\left\||x|^{m-1}\langle x\rangle^{-m-1-3 / 2} e^{i t\left(-\partial_{x}^{2}+h^{-2} V\right)} u_{0}\right\|_{L^{2}}^{2} d t \leqslant C h\left\|u_{0}\right\|_{L^{2}}^{2},
$$

and

$$
\int_{0}^{T}\left\|\langle x\rangle^{-3 / 2} e^{i t\left(-\partial_{x}^{2}+h^{-2} V\right)} u_{0}\right\|_{L^{2}}^{2} d t \leqslant C h^{1 /(m+1)}\left\|u_{0}\right\|_{L^{2}}^{2} .
$$

The dual versions of these estimates are given in the following Corollary.

Corollary 3.1. Let $V(x)=A^{-2}(x)+h^{2} V_{1}(x)$ as above. Then for any $T>0$, there exists a constant $C=C_{T}>0$ such that

$$
\left\|\int_{0}^{T}|x|^{m-1}\langle x\rangle^{-m-1-3 / 2} e^{-i t\left(-\partial_{x}^{2}+h^{-2} V\right)} f d t\right\|_{L^{2}}^{2} \leqslant C h\|f\|_{L_{T}^{2} L^{2}}^{2},
$$

and

$$
\left\|\int_{0}^{T}\langle x\rangle^{-3 / 2} e^{-i t\left(-\partial_{x}^{2}+h^{-2} V\right)} f d t\right\|_{L^{2}}^{2} \leqslant C h^{(1-m) /(m+1)}\|f\|_{L^{2}}^{2} .
$$

The purpose of these results is to demonstrate that there is perfect $1 / 2$ derivative local smoothing away from $x=0$, or local smoothing with either a loss in derivative or with a vanishing multiplier at $x=0$.

3.2. The endpoint Strichartz estimates. The endpoint Strichartz estimates are the $L_{T}^{2} L^{2^{\star}}\left[\right.$ estimates, where $2^{\star}$ is the Strichartz dual:

$$
1+\frac{n}{2^{\star}}=\frac{n}{2},
$$

which implies $2^{\star}=2 n /(n-2)$ for $n \geqslant 3$, and $2^{\star}=\infty$ if $n=2$.

We want to estimate $v$ in $L^{2^{\star}}(M)$, which we do using the following estimate due to Sogge [Sog86]:

Theorem 3 ( Sog86). Let $(M, g)$ be a d-dimensional compact Riemannian manifold without boundary, and let $-\Delta$ be the Laplace-Beltrami operator on $M$. If $\varphi_{j}$ are the eigenfunctions,

$$
-\Delta \varphi_{j}=\lambda_{j}^{2} \varphi_{j}
$$

with $0=\lambda_{1} \leqslant \lambda_{2} \leqslant \cdots$ the eigenvalues, then

$$
\left\|\varphi_{j}\right\|_{L^{2(d+1) /(d-1)}} \leqslant C \lambda_{j}^{(d-1) / 2(d+1)}\left\|\varphi_{j}\right\|_{L^{2}} .
$$

\footnotetext{
${ }^{1}$ Throughout this manuscript, we use the notation $L_{T}^{p} L^{q}=L^{p}([0, T]) L^{q}(M)$ to denote the local in time, global in space Strichartz norm.
} 
In particular, for the situation at hand,

$$
\begin{aligned}
& \|v\|_{L^{2^{\star}}(\mathbb{R}) L^{2^{\star}}\left(\mathbb{S}^{n-1}\right)}=\left\|H_{k} v\right\|_{L^{2^{\star}}(\mathbb{R}) L^{2^{\star}}\left(\mathbb{S}^{n-1}\right)}
\end{aligned}
$$

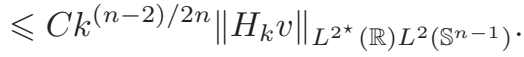

Now let $\Lambda_{k}$ be an index set for the $k$ th harmonic subspace $\mathcal{H}_{k}$ and write

$$
v(t, x, \theta)=\sum_{l \in \Lambda_{k}} v_{l k}(t, x) H_{l k}(\theta),
$$

where $H_{l k}$ are the orthonormal spherical harmonics in $\mathcal{H}_{k}$. Now if $p \geqslant 2,2 \leqslant q \leqslant 2^{*}$ $(q<\infty$ if $n=2)$, we have

$$
\begin{aligned}
\|v\|_{L_{T}^{p} L^{q}(M)} & \leqslant C k^{(n-2) / 2 n}\|v\|_{L_{T}^{p} L^{q}(\mathbb{R}) L^{2}\left(\mathbb{S}^{n-1}\right)} \\
& \leqslant C k^{(n-2) / 2 n}\left\|\left(\sum_{l \in \Lambda_{k}}\left|v_{l k}\right|^{2}\right)^{1 / 2}\right\|_{L_{T}^{p} L^{q}(\mathbb{R})},
\end{aligned}
$$

by Plancherel's theorem. We further estimate using Minkowski's inequality repeatedly:

$$
\begin{aligned}
\left\|\left(\sum_{l \in \Lambda_{k}}\left|v_{l k}\right|^{2}\right)^{1 / 2}\right\|_{L_{T}^{p} L^{q}(\mathbb{R})} & =\left(\int_{0}^{T}\left[\left(\int\left(\sum_{l}\left|v_{l k}\right|^{2}\right)^{q / 2} d x\right)^{2 / q}\right]^{p / 2} d t\right)^{1 / p} \\
& \leqslant C\left(\int_{0}^{T}\left[\sum_{l}\left(\int\left|v_{l k}\right|^{q} d x\right)^{2 / q}\right]^{p / 2} d t\right)^{1 / p} \\
& =C\left(\int_{0}^{T}\left[\left(\sum_{l}\left\|v_{l k}\right\|_{L^{q}}^{2}\right)^{1 / 2}\right]^{p} d t\right)^{1 / p} \\
& =C\left[\left(\int_{0}^{T}\left(\sum_{l}\left\|v_{l k}\right\|_{L^{q}}^{2}\right)^{p / 2} d t\right)^{2 / p}\right]^{1 / 2} \\
& \leqslant C\left[\sum_{l}\left(\int_{0}^{T}\left\|v_{l k}\right\|_{L^{q}}^{p} d t\right)^{2 / p}\right]^{1 / 2} \\
& =C\left(\sum_{l \in \Lambda_{k}}\left\|v_{l k}\right\|_{L_{T}^{p} L^{q}}^{2}\right)^{1 / 2} \cdot
\end{aligned}
$$

All told then we have

$$
\|v\|_{L_{T}^{p} L^{q}(M)} \leqslant C k^{(n-2) / 2 n}\left(\sum_{l \in \Lambda_{k}}\left\|v_{l k}\right\|_{L_{T}^{p} L^{q}}^{2}\right)^{1 / 2},
$$

where $v_{l k}(t, x)=\left\langle v(t, x, \cdot), H_{l k}\right\rangle_{L^{2}\left(\mathbb{S}^{n-1}\right)}$.

Using (2.3), we see that $v_{l k}$ satisfies the equation $\left(D_{t}+P_{k}\right) v_{l k}=0$, which is a 1-dimensional Schrödinger equation with potential. We want to estimate $v_{l k}$ in the $L_{T}^{2} L^{2^{\star}}(\mathbb{R})$ norm when $n \geqslant 3$, and in $L_{T}^{p} L^{q}$ Strichartz duals with $2 \leqslant q<\infty$ 
in dimension $n=2$. However, since we are now looking at a solution to a one dimensional Schrödinger equation, 2 and $2^{\star}$ are not Strichartz duals in 1 dimension. The Strichartz dual $p$ to $2^{\star}=2 n /(n-2)(n \geqslant 3)$ in one dimension satisfies

$$
\frac{2}{p}+\frac{1}{2^{\star}}=\frac{1}{2}
$$

or

$$
p=2 n .
$$

We therefore first use Hölder's inequality in $t$, with weights $n$ and $n /(n-1)$ respectively to get

$$
\begin{aligned}
\left\|v_{l k}\right\|_{L_{T}^{2} L^{2^{\star}}}^{2} & =\int_{0}^{T}\left\|v_{l k}\right\|_{L^{2^{\star}}}^{2} d t \\
& \leqslant T^{(n-1) / n}\left\|v_{l k}\right\|_{L_{T}^{2 n} L^{2^{\star}}}^{2} .
\end{aligned}
$$

In dimension $n=2$, we use $p>2,2 \leqslant q<\infty$ and the same weights in Hölder's inequality to get

$$
\begin{aligned}
\left\|v_{l k}\right\|_{L_{T}^{p} L^{q}}^{2} & =\int_{0}^{T}\left\|v_{l k}\right\|_{L^{2^{\star}}}^{2} d t \\
& \leqslant T^{1 / p}\left\|v_{l k}\right\|_{L_{T}^{2 n} L^{2^{\star}}}^{2} .
\end{aligned}
$$

We have the following proposition.

Proposition 3.2. Suppose $v_{l k}$ solves

$$
\left\{\begin{array}{l}
\left(D_{t}+P_{k}\right) v_{l k}=0, \\
\left.v_{l k}\right|_{t=0}=v_{l k}^{0},
\end{array}\right.
$$

where $v_{l k}^{0} \in H^{s}$ for some $s>0$. Then for any $T>0$, there exists a constant $C=C_{T}>0$ such that

$$
\left\|v_{l k}\right\|_{L_{T}^{2 n} L^{2^{\star}}} \leqslant C\left\|\langle k\rangle^{\beta} v_{l k}^{0}\right\|_{L^{2}} .
$$

That is, even though $v_{l k}$ solves a Schrödiner equation with a degenerate potential barrier, $v_{l k}$ nevertheless satisfies Strichartz estimates with an arbitrary $\beta>0$ loss. As a consequence, we have the following estimate on natural semiclassical time scales.

Corollary 3.3. Suppose $v$ solves (2.1) with initial data $v_{0}=v_{l k}^{0} H_{l k}$. Then for $\epsilon>0$ sufficiently small and $T=\epsilon k^{-2 /(m+1)}, v$ satisfies the Strichartz estimate

$$
\|v\|_{L_{T}^{2} L^{2^{\star}}} \leqslant C\left\|\langle k\rangle^{\eta+\beta} v_{0}\right\|_{L^{2}}
$$

where

$$
\eta=\frac{1}{2 n(m+1)}(m(n-2)-n) .
$$

Moreover, if $v_{l k}$ is a zonal spherical harmonic, this estimate is near-sharp, in the sense that no polynomial derivative improvement is true for every $\beta>0$.

Remark 3.4. This corollary shows that on natural semiclassical time scales the Strichartz estimates are improved. Indeed, in dimension $n=2$, there is a smoothing effect. The proof of the near-sharpness of this estimate is in Section 5.1. 
3.3. Proof of Proposition 2.2 and Corollary 3.3. Assuming Proposition 3.2 we have from (3.1) (in dimension $n \geqslant 3$ ):

$$
\begin{aligned}
\|v\|_{L_{T}^{2} L^{2^{\star}}(M)}^{2} & \leqslant C k^{(n-2) / n} \sum_{l \in \Lambda_{k}}\left\|v_{l k}\right\|_{L_{T}^{2} L^{2^{\star}}(\mathbb{R})}^{2} \\
& \leqslant C k^{(n-2) / n} T^{(n-1) / n} \sum_{l \in \Lambda_{k}}\left\|\langle k\rangle^{\beta} v_{l k}^{0}\right\|_{L^{2}(\mathbb{R})}^{2} \\
& \leqslant C T^{(n-1) / n}\left\|\langle k\rangle^{(n-2) / 2 n+\beta} v_{0}\right\|_{L^{2}(M)}^{2},
\end{aligned}
$$

by orthonormality, which is Proposition 2.2 and hence also Theorem 1. A similar computation using (3.1) holds when $n=2$, and $2 \leqslant q<\infty$.

For Corollary 3.3, the sum is over only one term, and $T \sim k^{-2 /(m+1)}$. Then in this case,

$$
\begin{aligned}
\|v\|_{L_{T}^{2} L^{2^{\star}}(M)}^{2} & \leqslant C k^{-2(n-1) / n(m+1)} k^{(n-2) / n}\left\|v_{l k}\right\|_{L^{2^{\star}}(\mathbb{R})}^{2} \\
& \leqslant(1+|k|)^{\frac{1}{n(m+1)}(m(n-2)-n)}\left\|\langle k\rangle^{\beta} v_{l k}^{0}\right\|_{L^{2}(M)}^{2} \\
& \leqslant C\left\|\langle k\rangle^{\eta+\beta} v_{0}\right\|_{L^{2}(M)}^{2},
\end{aligned}
$$

where $\eta$ is as in Corollary 3.3. A similar computation holds for $q<\infty$ in the case $n=2$.

\section{THE PARAMETRIX}

It remains to prove Proposition 3.2 For that purpose, in this section we construct a parametrix for the separated Schrödinger equation:

$$
\left\{\begin{array}{l}
\left(D_{t}+\left(-\partial_{x}^{2}+\lambda_{k}^{2} A^{-2}(x)+V_{1}(x)\right)\right) u=0, \\
\left.u\right|_{t=0}=u_{0} .
\end{array}\right.
$$

We rescale $h^{2}=\lambda_{k}^{-2}$ to get

$$
\left\{\begin{array}{l}
\left(D_{t}-\left(-\partial_{x}^{2}+h^{-2} A^{-2}(x)+V_{1}(x)\right)\right) u=0, \\
\left.u\right|_{t=0}=u_{0} .
\end{array}\right.
$$

Let $v(t, x)=u(h t, x)$, so that

$$
\left\{\begin{array}{l}
\left(h D_{t}-\left(-h^{2} \partial_{x}^{2}+A^{-2}(x)+h^{2} V_{1}(x)\right)\right) v=0, \\
\left.v\right|_{t=0}=u_{0} .
\end{array}\right.
$$

For the rest of this section, we consider the one-dimensional semiclassical Schrödinger equation with barrier potential:

$$
\left\{\begin{array}{l}
\left(h D_{t}+\left(-h^{2} \partial_{x}^{2}+V(x)\right)\right) v=0 \\
\left.v\right|_{t=0}=v_{0}
\end{array}\right.
$$

The potential $V(x)=A^{-2}(x)+h^{2} V_{1}(x)$ decays at $|x|=\infty$, is even, and the principal part $A^{-2}(x)$ has a degenerate maximum at $x=0$ with no other critical points. Denote $P=-h^{2} \partial_{x}^{2}+V(x)$.

Let us give a brief summary of the steps involved in the construction. We will use a WKB type approximation, although, since we are in dimension 1, we do not need a particularly good approximation. The first step is to approximate 
the solution away from the critical point at $(x, \xi)=(0,0)$. Since this is a nontrapping region, standard techniques can be used to construct a parametrix and prove Strichartz estimates on a timescale $t \sim h^{-1}$ for the semiclassical problem, or on a fixed timescale for the classical problem. A similar construction applies for energies away from the trapped set.

The remaining regions can be divided into an $h$-dependent strongly trapped region and a "transition region", where wave packets propagate, but not at a uniform rate. By restricting attention to a sufficiently small $h$-dependent neighbourhood of $(0,0)$, we can extend a semiclassical parametrix to a timescale $t \sim h^{(1-m) /(1+m)}$, which is a classical timescale of $h^{2 /(m+1)}$. We divide the transition region into a logarithmic number of $h$-dependent regions on which a similar parametrix construction works. Summing over all of these regions gives a parametrix construction and corresponding Strichartz estimates in a compact region in phase space with a logarithmic loss due to the number of summands in the transition region. These constructions and Strichartz estimates hold for a frequency dependent timescale $\sim h^{2 /(m+1)+\beta}$, $\beta>0$, or with a $\beta>0$ loss in derivative on timescale $\sim h^{2 /(m+1)}$. We then use the local smoothing estimate from CW11 to glue estimates on $\sim h^{-2 /(m+1)}$ time intervals to get the Strichartz estimates with a $\beta>0$ loss overall.

4.1. WKB expansion. We make the following WKB ansatz:

$$
v=h^{-1 / 2} \int e^{i \varphi(t, x, \xi) / h} e^{-i y \xi} B(t, x, \xi) u_{0}(y) d y d \xi,
$$

and compute

$$
\left(h D_{x}\right)^{2} v=\int e^{i \varphi(t, x, \xi) / h}\left(\left(\varphi_{x}\right)^{2} B-i h \varphi_{x x} B-2 i h \varphi_{x} B_{x}-h^{2} B_{x x}\right) u_{0}(y) d x d \xi,
$$

and

$$
h D_{t} v=\int e^{i \varphi(t, x, \xi) / h}\left(\varphi_{t} B-i h B_{t}\right) u_{0}(y) d y d \xi .
$$

In order to approximately solve the semiclassical Schrödinger equation for $v$, we use the WKB analysis. We begin by trying to construct $\varphi$ so that

$$
\left\{\begin{array}{l}
\varphi_{t}+\left(\varphi_{x}\right)^{2}+V(x)=0 \\
\left.\varphi\right|_{t=0}=x \xi
\end{array}\right.
$$

Given such $\varphi$, we solve the transport equations for the amplitude using a semiclassical expansion:

$$
B=\sum_{j \geqslant 0} h^{j} B_{j}(t, x, \xi)
$$

and

$$
-i h B_{t}-i h \varphi_{x x} B-2 i h \varphi_{x} B_{x}-h^{2} B_{x x}=0 .
$$

This amounts to solving:

$$
\left\{\begin{array}{l}
-B_{0, t}-2 \varphi_{x} B_{0, x}-\varphi_{x x} B_{0}=0, \\
-i B_{j, t}-i \varphi_{x x} B_{j}-2 i \varphi_{x} B_{j, x}-B_{j-1, x x}=0, j \geqslant 1 .
\end{array}\right.
$$


4.2. The partition of unity. In this subsection we construct the partition of unity which will be used to glue together the parametrices constructed in the following subsections. Let $\epsilon>0, \delta>0$ be sufficiently small, and $\omega>1$, all to be specified in the sequel. Let $\chi \in \mathcal{C}_{c}^{\infty}(\mathbb{R}), \chi(r) \equiv 1$ for $|r| \leqslant 1$, with support in $\{|r| \leqslant 2\}$ and assume $\chi^{\prime}(r) \leqslant 0$ for $r \geqslant 0$. For $\sigma>0$, let $\chi_{\sigma}(r)=\chi(r / \sigma)$. Let $\chi^{ \pm} \in \mathcal{C}^{\infty}(\mathbb{R})$, $\chi^{ \pm}(r) \equiv 1$ for $\pm r \gg 1, \chi^{ \pm}=0$ for $\pm r \geqslant 0$, and choose $\chi^{ \pm}$so that $1=\chi(r)+$ $\chi^{+}(r)+\chi^{-}(r)$, and denote also $\chi_{\sigma}^{ \pm}(r)=\chi^{ \pm}(r / \sigma)$. Choose also $\psi_{0}, \psi \in \mathcal{C}_{c}^{\infty}\left(\mathbb{R}_{+}\right)$ with $\psi_{0}(r) \equiv 1$ near $r=0$, and $\psi(r) \equiv 1$ in a neighbourhood of $r=\delta$ such that

$$
\sum_{0}^{N(h)} \psi\left(\omega^{j} x\right) \equiv 1 \text { for } x \in\left[\delta, 2 \epsilon h^{-1 /(m+1)}\right],
$$

and

$$
\psi_{0}(x)+\sum_{0}^{N(h)}\left(\psi\left(\omega^{j} x\right)+\psi\left(-\omega^{j} x\right)\right) \equiv 1 \text { for } x \in\left[-2 \epsilon h^{-1 /(m+1)}, 2 \epsilon h^{-1 /(m+1)}\right] .
$$

We remark for later use that we take, for example

$$
\psi\left(\omega^{j} x\right)=\left\{\begin{array}{l}
1, \text { for } \delta\left(\omega^{j}+\omega^{j-2}\right) \leqslant x \leqslant \delta\left(\omega^{j+1}-\omega^{j-1}\right) \\
0, \text { for } x \in\left[\delta\left(\omega^{j}-\omega^{j-2}\right), \delta\left(\omega^{j+1}+\omega^{j-1}\right)\right]^{\complement},
\end{array}\right.
$$

so that in particular

$$
\left|\partial_{x}^{k} \psi\left(\omega^{j} x\right)\right| \leqslant C_{k}(\delta \omega)^{k}\left(\omega^{-j k}\right) .
$$

We also observe this implies we need $N(h)$ sufficiently large that $\omega^{j} \sim h^{-1 /(m+1)}$, so that $N(h)=\mathcal{O}(\log (1 / h))$, with constants depending on $\delta, \omega$, and $m$.

We write

$$
e^{i t P / h}=L(t)+S(t):=\left(1-\chi_{\epsilon}(x)\right) e^{i t P / h}+\chi_{\epsilon}(x) e^{i t P / h}
$$

for the propagator cut off to large and small values of $x$ respectively. The set where the symbol $p=1$ contains the critical point $(0,0)$, so we further decompose into frequencies $\xi$ which lie above (respectively below) the set where $p=1$, and frequencies which are bounded:

$$
S(t)=S_{\mathrm{hi}}(t)+S_{\mathrm{lo}}(t),
$$

where

$$
S_{\mathrm{hi}}(t)=\mathbb{1}_{\left\{ \pm h D_{x} \geqslant 1-V(x)\right\}}\left(1-\chi_{\epsilon^{2}}((P-1))\right) S(t),
$$

and $S_{\mathrm{lo}}(t)=S(t)-S_{\mathrm{hi}}(t)$. We decompose yet again to

$$
S_{\mathrm{lo}}(t)=S_{\mathrm{lo}, 0,0}(t)+\sum_{j=0}^{N(h)}\left(S_{\mathrm{lo}, j,+}(t)+S_{\mathrm{lo}, j,-}(t)\right),
$$

where

$$
S_{\mathrm{lo}, 0,0}(t)=\psi_{0}\left(x / h^{1 /(m+1)}\right) S_{\mathrm{lo}}(t),
$$

and

$$
S_{\mathrm{lo}, j, \pm}(t)=\psi\left( \pm \omega^{j} x / h^{1 /(m+1)}\right) S_{\mathrm{lo}}(t) .
$$

The operators $S_{\mathrm{lo}, j, \pm}(t)$ are localized to bounded frequencies, and dyadic strips of size $h^{1 /(m+1)} \omega^{j}$. We require one further localization, which is to assume that the operators are also outgoing/incoming. Choose $\tilde{\chi} \in \mathcal{C}^{\infty}(\mathbb{R})$ so that $\tilde{\chi}(r)=1$ for $r \geqslant 1$ and $\tilde{\chi}(r)=0$ for $r \leqslant 0$. For $a, \gamma>0$ to be determined, let

$$
S_{\mathrm{lo}, j,+}^{ \pm}(t)=\tilde{\chi}\left(\left( \pm h D_{x}+a x^{m}\right) / \gamma x^{m}\right) S_{\mathrm{lo}, j,+}(t),
$$


and

$$
S_{\mathrm{lo}, j,-}^{ \pm}(t)=\tilde{\chi}\left(\left(\mp h D_{x}+a x^{m}\right) / \gamma x^{m}\right) S_{\mathrm{lo}, j,-}(t) .
$$

This has the effect of localizing in phase space to the sets where

$$
\pm \xi \geqslant-a x^{m}
$$

for $S_{\mathrm{lo}, j,+}^{ \pm}(t)$ and similarly for $S_{\mathrm{lo}, j,-}^{ \pm}(t)$. By the properties of $\tilde{\chi}$, we have

$$
S_{\mathrm{lo}, j,+}^{ \pm}(t)=S_{\mathrm{lo}, j,+}(t)
$$

microlocally on the set

$$
\left\{ \pm \xi \geqslant(\gamma-a) x^{m}\right\}
$$

If $a>\gamma$, these two sets clearly cover the remaining phase space, so if we can estimate each one of the operators above, we have estimated the entire propagator.

It is clear then that if we can prove, say, $\beta / 2>0$ loss Strichartz estimates for $S_{\mathrm{lo}, 0,0}(t)$, and for each $S_{\mathrm{lo}, j,+}^{+}$and $S_{\mathrm{lo}, j,-}^{-}(t)$ for $t \geqslant 0$, the Strichartz estimates follow for $S_{\mathrm{lo}, j,+}^{-}$and $S_{\mathrm{lo}, j,-}^{+}(t)$ by time reversal. We thus have to prove Strichartz estimates for each of these operators, as well as for $S_{\mathrm{hi}}(t)$ and $L(t)$, at which point we can sum up and take a $\operatorname{loss}$ of $\log (1 / h)+h^{-\beta / 2}<C h^{-\beta}$.

4.3. The parametrix for $L(t)$. We recall that the operator $L(t)$ is the propagator localized to large $|x|$. Then the operator $L(t)$ can be decomposed into $L^{+}(t)+L^{-}(t)$, supported where $\pm x>0$ respectively. Thus

$$
L^{+}(t)=\chi_{\epsilon}^{+}(x) e^{i t P / h} \text {. }
$$

By a $T T^{*}$ argument (see $\underline{\text { KT98 }}$ ), in order to show $L^{+}: L^{2} \rightarrow L_{T}^{p} L^{q}$, it suffices to estimate

$$
L^{+}(t)\left(L^{+}\right)^{*}(s): L^{1} \rightarrow L^{\infty},
$$

but

$$
L^{+}(t)\left(L^{+}\right)^{*}(s)=\chi_{\epsilon}^{+}(x) e^{i(t-s) P / h} \chi_{\epsilon}^{+}(x) .
$$

That is, we need only construct a parametrix supported for $x \geqslant \epsilon$, and for initial data supported for $x \geqslant \epsilon$.

Lemma 4.1. There exist constants $C>0$ and $\alpha>0$ such that for any $u_{0} \in L^{1} \cap L^{2}$, we have

$$
\left\|L^{+}(t)\left(L^{+}\right)^{*}(s) u_{0}\right\|_{L_{x}^{\infty}} \leqslant C(|t-s| h)^{-1 / 2}\left\|u_{0}\right\|_{L^{1}},
$$

for $|t|,|s| \leqslant \alpha h^{-1}$. As a consequence,

$$
\left\|L(t) u_{0}\right\|_{L_{\alpha h^{-1}}^{p} L^{q}} \leqslant h^{-1 / p}\left\|u_{0}\right\|_{L^{2}}
$$

for

$$
\frac{2}{p}+\frac{1}{q}=\frac{1}{2}, 2 \leqslant q<\infty
$$

Proof. The proof is simply to observe that $L^{+}(t)\left(L^{+}\right)^{*}(s)$ is equal to a non-trapping cut-off propagator, and hence obeys a strong dispersion and perfect Strichartz estimates according to BT08.

To see this, let

$$
\tilde{A}(x)^{-2}=\chi(x / \epsilon) x^{-2}+(1-\chi(x / \epsilon)) A^{-2} .
$$

The function $\tilde{A}$ agrees with $A$ for large $x$ and agrees with $x$ for small $x$. Then

$$
\tilde{g}=d x^{2}+\tilde{A}^{2}(x) d \theta^{2}, \quad x \geqslant 0
$$


is an asymptotically Euclidean metric, which agrees with the Euclidean metric near $x=0$. In fact, since $g$ was a short-range perturbation of the Euclidean metric as $x \rightarrow+\infty$, so is $\tilde{g}$. In addition, we claim that for $\epsilon>0$ sufficiently small, $\tilde{g}$ is a non-trapping perturbation of the Euclidean metric. To see this, we examine the geodesic equations. Let $\tilde{p}=\xi^{2}+\tilde{A}^{-2}(x) \eta^{2}$, and compute the geodesic equations:

$$
\left\{\begin{array}{l}
\dot{x}=2 \xi, \\
\dot{\xi}=2 \tilde{A}^{\prime} \tilde{A}^{-3} \eta^{2}, \\
\dot{\theta}=2 \tilde{A} \eta, \\
\dot{\eta}=0 .
\end{array}\right.
$$

Consider a unit speed geodesic with $\tilde{p} \equiv 1$. Since $\eta$ remains constant, then either $\eta=0$, in which case $\xi= \pm 1$ and $x \rightarrow \pm \infty$ uniformly, or $\eta \neq 0$. If $\xi \equiv 0$, then necessarily $\left(\tilde{A}^{-2}(x)\right)^{\prime}=0$ and $x$ is stationary, but

$$
\left(\tilde{A}^{-2}(x)\right)^{\prime}=-2 \chi(x / \epsilon) x^{-3}-2(1-\chi(x / \epsilon)) A^{\prime} A^{-3}+\epsilon^{-1} \chi^{\prime}(x \epsilon)\left(x^{-2}-A^{-2}\right) .
$$

But $A^{\prime}>0$ away from $x=0, x^{-2} \gg A^{-2}$ for $x>0$ sufficiently small, and $\chi^{\prime} \leqslant 0$ for $x>0$ implies $\left(\tilde{A}^{-2}(x)\right)^{\prime}<0$ for $x>0$. Hence there are no parallel periodic geodesics.

It remains to show that every other trajectory escapes to infinity. But since $\dot{\xi} \geqslant c^{-1} x^{-3} \eta^{2}$, comparing to the non-trapping conic metric with

$$
\left\{\begin{array}{l}
\dot{x}=2 \xi, \\
\dot{\xi}=c^{-1} x^{-3} \eta^{2}
\end{array}\right.
$$

implies that every other trajectory is non-trapped. Then following Bouclet-Tzvetkov BT08, we get that

$$
L^{+}: L^{2} \rightarrow L_{\alpha h^{-1}}^{p} L^{q}, \alpha>0,
$$

is a bounded operator for $(p, q)$ in the specified range. A similar estimate holds for $L^{-}$, and hence $L$, and hence for any $\epsilon>0$ sufficiently small, we can construct a parametrix to get perfect Strichartz estimates for $|x| \geqslant \epsilon$.

4.4. The parametrix for $S_{\mathbf{h i}}(t)$. The operator $S_{\mathrm{hi}}(t)$ is the propagator localized to small $|x| \leqslant 2 \epsilon$ and high frequencies $|P-1| \geqslant \epsilon^{2}$, and $\pm \xi \geqslant 1-V(x)$. In order to estimate $S_{\mathrm{hi}}(t)$, we employ a similar argument. We first decompose $S_{\mathrm{hi}}(t)=$ $S_{\mathrm{hi}}^{+}(t)+S_{\mathrm{hi}}^{-}(t)$ into a part supported in $\pm \xi>0$. The point of the next lemma is that singularities propagate out of this region quickly, depending on the initial frequency.

Lemma 4.2. There exist constants $\alpha, \kappa>0$ such that

$$
\chi^{+}\left(|t-s| h D_{x} / \kappa \epsilon\right) S_{h i}^{+}(t)\left(S_{h i}^{+}\right)^{*}(s)=\mathcal{O}\left(h^{\infty}\right)
$$

in any seminorm, provided $|t|,|s| \leqslant \alpha h^{-1}$

There exist constants $C>0$ and $\alpha>0$ such that for any $u_{0} \in L^{1} \cap L^{2}$, we have

$$
\left\|S_{h i}^{+}(t)\left(S_{h i}^{+}\right)^{*}(s) u_{0}\right\|_{L_{x}^{\infty}} \leqslant C(|t-s| h)^{-1 / 2}\left\|u_{0}\right\|_{L^{1}},
$$

for $|t|,|s| \leqslant \alpha h^{-1}$. As a consequence,

$$
\left\|S_{h i}(t) u_{0}\right\|_{L_{\alpha h^{-1}}^{p} L^{q}} \leqslant C h^{-1 / p}\left\|u_{0}\right\|_{L^{2}}
$$


for

$$
\frac{2}{p}+\frac{1}{q}=\frac{1}{2}, 2 \leqslant q<\infty .
$$

Proof. As usual, we consider the Hamiltonian system associated to $p$ :

$$
\left\{\begin{array}{l}
\dot{x}=2 \xi, \\
\dot{\xi}=-V^{\prime}(x), \\
x(0)=y, \\
\xi(0)=\eta,
\end{array}\right.
$$

where now $|x|,|y| \leqslant 2 \epsilon$ and $\eta \geqslant \epsilon$. Then a simple computation shows that in this region $\left|V^{\prime}(x)\right|=\mathcal{O}\left(\epsilon^{2 m-1}\right)$ and $\left|V^{\prime \prime}(x)\right|=\mathcal{O}\left(\epsilon^{2 m-2}\right)$. Hence if $t=\mathcal{O}(1)$, we have

$$
\xi=\eta+\mathcal{O}\left(\epsilon^{2 m-1}\right)=\eta\left(1+\mathcal{O}\left(\epsilon^{2 m-2}\right)\right),
$$

since $\eta \geqslant \epsilon$. Hence

$$
\dot{x}=2 \eta\left(1+\mathcal{O}\left(\epsilon^{2 m-2}\right)\right),
$$

so that

$$
x=y+2 \operatorname{t\eta }\left(1+\mathcal{O}\left(\epsilon^{2 m-2}\right)\right),
$$

provided $t=\mathcal{O}(1)$. This implies in particular, that for any $|t| \geqslant C \epsilon / \eta$, we will have $|x| \geqslant 2 \epsilon$, so that we have propagated out of the region of interest. Again, by virtue of a $T T^{*}$ argument, we are interested in both initial data and parametrix localized in $|x| \leqslant 2 \epsilon, \xi \geqslant \epsilon$, so we need only check the estimates on the phase function for $|t| \leqslant C \epsilon / \eta$.

We check the invertibility of the map $y \mapsto x(t)$ :

$$
\begin{aligned}
\sup _{|t| \leqslant C \epsilon / \eta}\left|\frac{\partial x}{\partial y}(t)\right| & \leqslant 1+2 \int_{0}^{C \epsilon / \eta}(C \epsilon / \eta-s)\left|V^{\prime \prime}(x)\right|\left|\frac{\partial x}{\partial y}(s)\right| d s \\
& \leqslant 1+\mathcal{O}\left(\epsilon^{2} / \eta^{2}\right) \mathcal{O}\left(\epsilon^{2 m-2}\right) \sup _{|s| \leqslant C \epsilon / \eta}\left|\frac{\partial x}{\partial y}(s)\right|
\end{aligned}
$$

which implies

$$
\sup _{|t| \leqslant C \epsilon / \eta}\left|\frac{\partial x}{\partial y}(t)\right| \leqslant 1+\mathcal{O}\left(\epsilon^{2 m-2}\right) .
$$

Similarly we compute the lower bound:

$$
\begin{aligned}
\inf _{|t| \leqslant C \epsilon / \eta}\left|\frac{\partial x}{\partial y}(t)\right| & \geqslant 1-2 \int_{0}^{C \epsilon / \eta}(C \epsilon / \eta-s)\left|V^{\prime \prime}(x)\right|\left|\frac{\partial x}{\partial y}(s)\right| d s \\
& \geqslant 1-\mathcal{O}\left(\epsilon^{2} / \eta^{2}\right) \mathcal{O}\left(\epsilon^{2 m-2}\right) \sup _{|s| \leqslant C \epsilon / \eta}\left|\frac{\partial x}{\partial y}(s)\right| \\
& \geqslant 1-\mathcal{O}\left(\epsilon^{2 m-2}\right),
\end{aligned}
$$

using our previously computed upper bound. Hence in the range in which we are interested, $\partial x / \partial y$ is uniformly bounded above and below by a constant, provided $\epsilon>0$ is chosen sufficiently small.

It is now a routine computation to construct the WKB amplitude and compute the dispersive estimate for $|t| \leqslant C \epsilon / \eta$. After that time, the $h$-wavefront set of a solution is outside the support of the cutoffs in $S_{\mathrm{hi}}(t)$, so that any parametrix approximation is $\mathcal{O}\left(h^{\infty}\right)$. Summing over $\mathcal{O}\left(h^{-1}\right)$ such parametrices yields the dispersive estimate for $|t| \leqslant \alpha h^{-1}$, and the associated Strichartz estimates. 
A similar computation works for $S_{\mathrm{hi}}(t)$ localized to $\xi \leqslant-\epsilon$, which proves the lemma for $S_{\mathrm{hi}}(t)$.

4.5. The parametrix for $S_{\mathrm{lo}, 0,0}(t)$. The operator $S_{\mathrm{lo}, 0,0}(t)$ is the propagator localized to small frequencies $|P-1| \leqslant \epsilon^{2}$ or $|P-1| \geqslant \epsilon^{2}$ with $|\xi| \leqslant 1-V(x)$, as well as localized to a small $h$-dependent spatial neighbourhood $|x| \leqslant \delta h^{1 /(m+1)}$. This is the region which contains the trapping. We observe that all of the $S_{\text {lo }}$ operators have $|x| \leqslant \epsilon$, which implies in addition that $|\xi| \leqslant 2 \epsilon$, say.

We are now interested in constructing a parametrix in the set $\left\{|x| \leqslant \delta h^{1 /(m+1)},|\xi| \leqslant\right.$ $\epsilon\}$. For this, we use the following $h$-dependent scaling operator:

$$
T_{h} u(t, x)=h^{-1 /(m+1)} u\left(h^{(m-1) /(m+1)} t, h^{-1 /(m+1)} x\right) .
$$

The purpose of the prefactor of $h^{-1 /(m+1)}$, different from the usual scaling prefactor, is to ensure that $\left\|T_{h} u\right\|_{L_{x}^{1}}=\|u\|_{L_{x}^{1}}$, since in our final dispersion estimate, this is how the initial data will be measured. We compute:

$$
\begin{aligned}
T_{h}^{-1}\left(h D_{t}-h^{2} \partial_{x}^{2}+V(x)\right) T_{h} & =\left(h^{(m-1) /(m+1)} h D_{t}-h^{-2 /(m+1)} h^{2} \partial_{x}^{2}+V\left(h^{1 /(m+1)} x\right)\right) \\
& =h^{2 m /(m+1)}\left(D_{t}-\partial_{x}^{2}+\widetilde{V}(x ; h)\right),
\end{aligned}
$$

where

$$
\widetilde{V}(x ; h)=h^{-2 m /(m+1)} V\left(h^{1 /(m+1)} x\right) .
$$

Remark 4.3. Similar to the the techniques in the paper [CW11], conjugation by the scaling operator $T_{h}$ is an inhomogeneous "blowup" procedure. However, the blowdown map $\mathcal{B}$ is now time-dependent and takes the form

$$
\mathcal{B}(t, \tau, x, \xi)=\left(h^{(1-m) /(m+1)} t, h^{2 m /(m+1)} \tau, h^{1 /(m+1)} x, h^{m /(m+1)} \xi\right) .
$$

That is, we are blowing up the $(\tau, x, \xi)$ coordinates and blowing down the $t$ coordinate at the same time. Observe that the blowdown in $t$ does not cause a problem with the calculus since the operator $P$ is independent of $t$. Then indeed, according to the calculus developed in [CW11], $\sigma_{h}(P)=\tau+\xi^{2}+V(x)$ in the $h$ calculus, while $T_{h}^{-1} P T_{h}$ has symbol

$$
\tilde{p}_{1}=\left(h^{2 m /(m+1)} \tau\right)+\left(h^{m /(m+1)} \xi\right)^{2}+V\left(h^{1 /(m+1)} x\right)
$$

in the 1-calculus, or scale-invariant calculus. Factoring out the $h^{2 m /(m+1)}$ as above results in a singular symbol in the scale-invariant calculus (see Figure2). However, the special structure of $V$ allows us to construct a reasonable parametrix where $V^{\prime}$ is extremely small, and where $V^{\prime}$ is large, wave packets propagate away in a controlled fashion. This is made rigorous in the following constructions.

Denote $\widetilde{P}=D_{t}-\partial_{x}^{2}+\widetilde{V}(x ; h)$, where

$$
\widetilde{V}(x ; h)=h^{-2 m /(m+1)} V\left(h^{1 /(m+1)} x\right) .
$$

We break the parametrix construction into two sets, where $\widetilde{V}^{\prime}$ is small (and hence this region contains the trapping), and where $\widetilde{V}^{\prime}$ is large, which we reserve for the next subsections where we estimate $S_{\mathrm{lo}, j, \pm}^{ \pm}(t)$.

We want to now construct a parametrix for $\widetilde{P}$ on the set

$$
\left\{|x| \leqslant \delta,|\xi| \leqslant 2 h^{-m /(m+1)},|t| \leqslant 1\right\},
$$




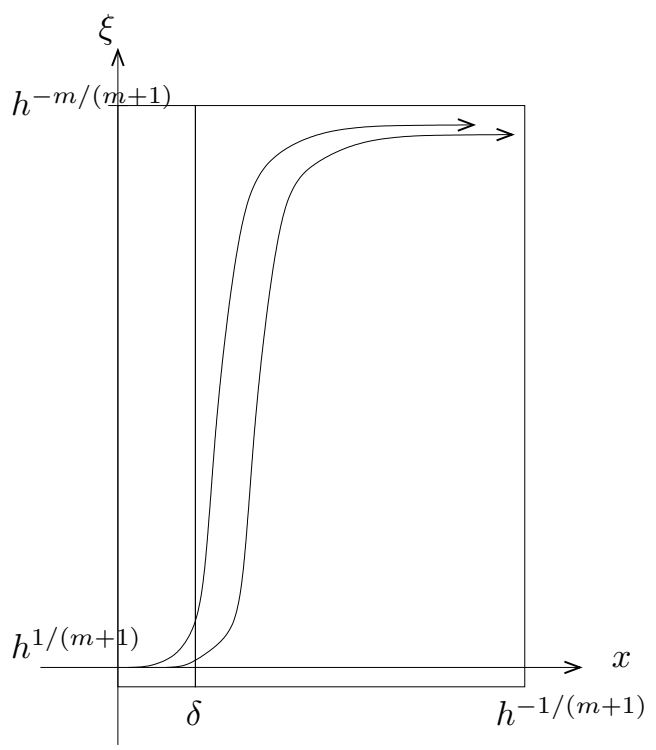

Figure 2. The phase plane in the blown up coordinates. The invariant curves are given by $L^{\zeta}=\left\{\xi=\sqrt{\zeta^{2}-\widetilde{V}(x)}\right.$, with $\zeta^{2} \geqslant$ $h^{-2 m /(m+1)}$. The boxes represent the $h$-wavefront set of a wave packet after rescaling $(x, \xi) \mapsto\left(h^{-1 /(m+1)} x, h^{-m /(m+1)} \xi\right)$, but in the 1-calculus.

but in the 1-calculus (scale-invariant). Then if $w(t, x)$ is such a parametrix, $v(t, x)=$ $T_{h} w(t, x)$ is a parametrix for $P$ on the set $\left\{|x| \leqslant \delta h^{1 /(m+1)},|\xi| \leqslant \epsilon,|t| \leqslant h^{(1-m) /(1+m)}\right\}$, as required.

Lemma 4.4. There exists $\alpha>0$ and a phase function $\varphi(t, x, \eta)$ satisfying

$$
\left\{\begin{array}{l}
\varphi_{t}+\varphi_{x}^{2}+\widetilde{V}(x ; h)=0, \\
\varphi(0, x, \eta)=x \eta
\end{array}\right.
$$

for $|x| \leqslant \delta,|\eta| \leqslant 2 \epsilon h^{-m /(m+1)}$, and $|t| \leqslant \alpha$.

We further have

$$
\varphi_{\eta \eta} \sim 2 t(1+\mathcal{O}(t))
$$

and

$$
\varphi_{x x}=\mathcal{O}\left(t x^{2 m-2}\right)
$$

for $|t| \leqslant \alpha$.

Proof. The proof is by the usual Hamiltonian method. We consider $q=\xi^{2}+\widetilde{V}(x ; h)$ and the Hamiltonian system associated to $q$ :

$$
\left\{\begin{array}{l}
\dot{x}=2 \xi, \\
\dot{\xi}=-\partial_{x} \widetilde{V}(x ; h), \\
x(0)=y, \\
\xi(0)=\eta .
\end{array}\right.
$$


Now the potential $\widetilde{V}(x ; h)$ has been computed above, and satisfies

$$
\begin{aligned}
-\partial_{x} \widetilde{V}(x ; h)= & -\partial_{x}\left(h^{-2 m /(m+1)}\left(1+\left(h^{1 /(m+1)} x\right)^{2 m}\right)^{-1 / m}+h^{2 /(m+1)} V_{1}\left(h^{1 /(m+1)} x\right)\right) \\
= & 2 h^{-2 m /(m+1)} h^{1 /(m+1)}\left(h^{1 /(m+1)} x\right)^{2 m-1}\left(1+\left(h^{1 /(m+1)} x\right)^{2 m}\right)^{-1 / m-1} \\
& +\mathcal{O}\left(h^{3 /(m+1)}\left(\left(h^{1 /(m+1)} x\right)^{2 m-3}\right) .\right.
\end{aligned}
$$

For $|x| \leqslant \delta$ this derivative is bounded, and has the same sign as $x$. Let us denote $B(x)=-\partial_{x} \widetilde{V}(x ; h)$ to avoid cumbersome notation.

In order to apply the usual Hamilton-Jacobi theory, we need to show that $\partial x / \partial y$ is uniformly bounded above and below by positive constants on some interval $|t| \leqslant$ $\alpha$, so that we can invert the transformation $y \mapsto x(t)$ to get $y=y(t, x)$. Then using $(x, \eta)$ as coordinates instead of $(y, \eta)$ proves the first part of the Lemma. We write

$$
x(t)=y+2 t \eta+\int_{0}^{t}(t-s) B(x(s)) d s,
$$

and compute

$$
\frac{\partial x}{\partial y}(t)=1+\int_{0}^{t}(t-s) B^{\prime}(x(s)) \frac{\partial x}{\partial y}(s) d s .
$$

We know

$$
\frac{\partial x}{\partial y}(0)=1
$$

and $B^{\prime}(x)$ is non-negative in a neighbourhood of $x=0$, so the integral in the above expression is positive for $|x| \leqslant \delta$ and $|t| \leqslant \alpha$ sufficiently small. Further, $B^{\prime}$ is bounded for $|x| \leqslant \delta$, so the integral expression is also bounded above for $|t| \leqslant \alpha$. Hence by restricting $|x|$ and $|t|$ to fixed, bounded ranges, we conclude the map sending $y \mapsto x(t)$ is invertible, and this completes the proof of the first assertion.

We observe that, by construction, $\varphi_{\eta}(t, x, \eta)=y$, so that to compute $\varphi_{\eta \eta}$, we need to compute

$$
\frac{\partial y}{\partial \eta}=\frac{\partial y}{\partial x} \frac{\partial x}{\partial \eta}
$$

We have already shown that $\partial y / \partial x$ is bounded above and below for $|t| \leqslant \alpha$, so we compute

$$
\begin{aligned}
\frac{\partial x}{\partial \eta} & =2 t+\int_{0}^{t}(t-s) B^{\prime}(x(s)) \frac{\partial x}{\partial \eta}(s) d s \\
& =2 t+\mathcal{O}\left(t^{2}\right) \sup \frac{\partial x}{\partial \eta} .
\end{aligned}
$$

This implies

$$
\sup _{|t| \leqslant \alpha} \frac{\partial x}{\partial \eta}(t) \leqslant 2 t(1+\mathcal{O}(t)) .
$$

Plugging this into the integral expression above yields

$$
\inf _{|t| \leqslant \alpha} \frac{\partial x}{\partial \eta} \geqslant 2 t\left(1+\mathcal{O}\left(t^{3}\right)\right) \text {. }
$$

Finally, since the intertwining relation gives $\varphi_{x}(t, x, \eta)=\xi$, we have

$$
\varphi_{x x}=\partial_{y} \xi \partial_{x} y
$$


in the notation above. We have already shown that $\partial_{x} y$ is bounded above and below by a positive constant for $|t| \leqslant \alpha$, so we just need to compute

$$
\begin{aligned}
\partial_{y} \xi & =\partial_{y}\left(\eta-\int_{0}^{t} \widetilde{V}^{\prime}(x(s)) d s\right) \\
& =-\int_{0}^{t} \widetilde{V}^{\prime \prime}(x(s)) \partial_{y} x(s) d s \\
& =\mathcal{O}\left(t x^{2 m-2}\right) .
\end{aligned}
$$

This is the last assertion in the Lemma.

We now construct the amplitude for the parametrix for the operator $S_{\mathrm{lo}, 0,0}(t)$. This, combined with Lemma 4.4 will be used to compute a dispersion estimate, resulting in a Strichartz estimate. The problem is that, since we are working in a marginal calculus, the error terms in our parametrix are just too large. For example, the error term $\varphi_{x x} \sim t x^{2 m-2}$ computed in Lemma 4.4 rescales as

$$
T_{h} \varphi_{x x} \sim h^{(m-1) /(m+1)} t h^{-(2 m-2) /(m+1)} x^{2 m-2} \sim h^{(1-m) /(1+m)} t x^{2 m-2} .
$$

This operator, when composed with the appropriate oscillatory integral, yields an $L^{2}$ bounded operator for each $t,|t| \leqslant h^{(1-m) /(m+1)}$. However, to apply an energy estimate or a local smoothing estimate, we either have to integrate in time (now an interval of length $\sim h^{(1-m) /(1+m)}$ ), or pull out a factor of $x^{m-1}$ to apply Theorem 2. In either case, we lose a factor of $h^{(1-m) / 2(m+1)}$. Hence at this point we must accept an $\beta>0$ loss in regularity by restricting our attention to a slightly smaller time interval. Then the "lower order" terms in the amplitude construction will actually gain powers of $h$.

We are interested in constructing a parametrix for the operator $S_{\mathrm{lo}, 0,0}(t) S_{\mathrm{lo}, 0,0}^{*}(s)$. We have constructed a phase function $\varphi(t, x, \xi)$ in rescaled coordinates, assuming appropriate microlocal cutoffs. That is, we have constructed the appropriate phase functions to approximate the operators

$$
T_{h}^{-1} S_{\mathrm{lo}, 0,0}(t) S_{\mathrm{lo}, 0,0}^{*}(s)=T_{h}^{-1} S_{\mathrm{lo}, 0,0}(t-s) \chi_{\star},
$$

where $\chi_{\star}$ is the appropriate microlocal cutoff. We have not yet computed the amplitude. Recalling the transport equations in the $h$-calculus (4.2), the transport equations for the amplitude $B$ in the rescaled 1-calculus coordinates become

$$
D_{t} B+2 \varphi_{x} D_{x} B-i \varphi_{x x} B-\partial_{x}^{2} B=0 .
$$

The standard technique here is to guess an asymptotic series, however, there is no small parameter, so we instead modify our ansatz to take advantage of the Frobenius theorem.

That is, the Frobenius theorem guarantees the existence of a function $\Gamma(t, x)$, depending implicitly on the frequency $\xi$, satisfying

$$
\left\{\begin{array}{l}
\partial_{t} \Gamma+2 \varphi_{x} \partial_{x} \Gamma=0, \\
\Gamma(0, x)=x
\end{array}\right.
$$


We then construct $B=\sum_{j=0}^{K} B_{j}$ for sufficiently large $K$ to be determined (independent of $h$ ) with

$$
\left\{\begin{array}{l}
B_{0} \equiv 1 \\
B_{j}=-\left.\int_{0}^{t} \varphi_{x x} B_{j-1}\right|_{(s, \Gamma(t-s, x))}+\left.i B_{j-1, x x}\right|_{(s, \Gamma(t-s, x))} .
\end{array}\right.
$$

An induction argument shows that $B_{j}=\mathcal{O}\left(t^{j}\right)$ for each $j$, since we are in the scale invariant calculus.

Then

$$
w(t, x)=(2 \pi)^{-1} \int e^{i \varphi(t, x, \xi)-i y \xi} B(t, x, \xi) \chi_{\star}\left(y, D_{y}\right)^{*} w_{0}(y) d y d \xi
$$

solves

$$
\left\{\begin{array}{l}
\widetilde{P} w=\widetilde{E}, \\
w(0, x)=\chi_{\star}\left(x, D_{x}\right)^{*} w_{0}(x),
\end{array}\right.
$$

where

$$
\chi_{\star}=T_{h}^{-1} \psi_{0}\left(x / h^{1 /(m+1)}\right)\left(1-\mathbb{1}_{\left\{ \pm h D_{x} \geqslant 1-V(x)\right\}}\left(1-\chi_{\epsilon^{2}}((P-1))\right)\right) \chi_{\epsilon}(x) T_{h}
$$

is the appropriate microlocal cutoff, and the equation is understood to make sense for $|t| \leqslant \alpha$. Here, the error $\widetilde{E}$ is given by

$$
\widetilde{E}=(2 \pi)^{-1} \int e^{i \varphi(t, x, \xi)-i y \xi}\left(-\partial_{x}^{2} B_{K}-i \varphi_{x x} B_{K}\right) \chi^{*}\left(y, D_{y}\right) w_{0}(y) d y d \xi
$$

That is, $\widetilde{E}$ is an oscillatory integral operator with the same phase function as $w$, and amplitude $A(t, x, \xi)$ satisfying

$$
\left|\partial_{x}^{k} \partial_{\xi}^{l} A\right| \leqslant C_{k l} t^{K}
$$

and hence, according to the next Lemma, satisfies

$$
\|\widetilde{E}\|_{L_{x}^{2}}=\mathcal{O}\left(t^{K}\right)\left\|\chi^{*} w_{0}\right\|_{L^{2}} .
$$

Lemma 4.5. Suppose $\Gamma(t, x, \xi) \in \mathcal{C}_{b}^{\infty} \mathcal{S}_{0,0}$ is a smooth family of symbols with bounded derivatives, and let $F(t), 0 \leqslant t \leqslant \alpha$ be the operator defined by

$$
F(t) g(x)=\int e^{i \varphi(t, x, \xi)-i y \xi} \Gamma(t, x, \xi) \chi_{\star}\left(y, D_{y}\right) g(y) d y d \xi,
$$

where $\varphi$ is the phase function constructed above and $\chi_{\star}$ is the appropriate microlocal cutoff. Then

$$
\sup _{0 \leqslant t \leqslant \alpha}\|F(t) g\|_{L^{2}} \leqslant C\|g\|_{L^{2}} .
$$

Proof. Let us work microlocally to avoid continually using microlocal cutoffs, and therefore assume the appropriate microlocal concentration. The $L^{2}$ boundedness of $F(t)$ is equivalent to the $L^{2}$ boundedness of $F(t)^{*}$, which follows from the $L^{2}$ boundedness of $F(t) F(t)^{*}$. The operator $F(t) F(t)^{*}$ is easily seen to have integral kernel

$$
K=\int e^{i \varphi(t, x, \xi)-i \varphi\left(t, x^{\prime}, \xi\right)} \Gamma(t, x, \xi) \bar{\Gamma}\left(t, x^{\prime}, \xi\right) d \xi,
$$

where again we are implicitly assuming appropriate microlocal cutoffs.

By stationary phase, this integral kernel has singularities when

$$
\partial_{\xi}\left(\varphi(t, x, \xi)-\varphi\left(t, x^{\prime}, \xi\right)\right)=0,
$$


which is when (using the notations from the phase construction)

$$
y(t, x, \xi)-y\left(t, x^{\prime}, \xi\right)=0 .
$$

Let us assume that $x \geqslant x^{\prime}$, so that we want to compute where

$$
\left(x-x^{\prime}\right)\left(\left.\partial_{x} y\right|_{x}+\mathcal{O}\left(\partial_{x}^{2} y\left(x-x^{\prime}\right)\right)\right) .
$$

Now due to the microlocal cutoffs $\chi_{\star}$, we have that $x$ and $x^{\prime}$ are both small. By the inverse function theorem and the boundedness of $\partial_{x} y$, we need to estimate $\partial_{y}^{2} x$ in the Hamiltonian systems used to construct the phase functions. We compute

$$
\partial_{y}^{2} x=-\int_{0}^{t}\left(\widetilde{V}^{\prime \prime \prime}(x)\left(\partial_{y} x\right)^{2}+\widetilde{V}^{\prime \prime}(x) \partial_{y}^{2} x\right) d s,
$$

and estimating the first term by $c$ for a small constant $c$ and solving for $\sup \partial_{y}^{2} x$ shows that

$$
\left|\mathcal{O}\left(\partial_{x}^{2} y\left(x-x^{\prime}\right)\right)\right| \leqslant c^{\prime}
$$

where $c^{\prime}>0$ is a small constant depending on our previous choices of $\epsilon, \delta$, and $\omega$.

Iterating this argument for other powers of $\left(x-x^{\prime}\right)$ shows that the singularities of the integral kernel lie on the diagonal $\left|x-x^{\prime}\right|=0$, so the integral kernel defines a 0 order pseudodifferential operator with symbol in the class $\mathcal{S}_{0,0}$. By the CalderónVaillancourt theorem, the $L^{2}$ boundedness is established.

If we now take $v=T_{h} w$, we see

$$
\begin{aligned}
P v & =T_{h} T_{h}^{-1} P T_{h} w \\
& =h^{2 m /(m+1)} T_{h} \widetilde{P} w \\
& =E,
\end{aligned}
$$

with initial conditions

$$
v(0, x)=T_{h} w(0, x)
$$

and where

$$
E=h^{2 m /(m+1)} T_{h} \widetilde{E} .
$$

A simple computation shows that $\left\|T_{h} f\right\|_{L^{2}}=h^{-1 / 2(m+1)}\|f\|_{L^{2}}$, so that if we now restrict attention to the smaller (rescaled) time interval

$$
0 \leqslant t \leqslant \alpha h^{(1-m) /(m+1)+\beta}
$$

for some small fixed $\beta>0$, we have

$$
\begin{aligned}
\sup _{0 \leqslant t \leqslant \alpha h^{(1-m) /(m+1)+\beta}}\|E\|_{L^{2}} & =h^{(4 m-1) / 2(m+1)} \sup _{0 \leqslant t \leqslant \alpha h^{\beta}}\|\widetilde{E}\|_{L^{2}} \\
& \leqslant C h^{(4 m-1) / 2(m+1)} h^{\beta K}\left\|\chi_{\star}^{*} w_{0}\right\|_{L^{2}} \\
& \leqslant C h^{2 m /(m+1)} h^{\beta K}\left\|\chi_{\star}^{*} v_{0}\right\|_{L^{2}} .
\end{aligned}
$$

Here, in the above computations, we have suppressed the variables of the microlocal cutoffs $\chi_{\star}$, which are understood to be evaluated in the phase space variables of the appropriate scale.

The following lemma contains the dispersion and Strichartz estimates for the operators $S_{\mathrm{lo}, 0,0}(t)$. 
Lemma 4.6. The parametrix $v(t, x)$ satisfies the dispersion estimate

$$
\left\|\chi_{\star} v\right\|_{L^{\infty}} \leqslant C(h t)^{-1 / 2}\left\|\tilde{\chi} v_{0}\right\|_{L^{1}}
$$

where $0<t \leqslant \alpha h^{(1-m) /(1+m)}$, as well as the corresponding Strichartz estimate

$$
\|v\|_{L_{\alpha h}^{p}(1-m) /(1+m)} L^{q} \leqslant C h^{-1 / p}\left\|\chi v_{0}\right\|_{L^{2}}
$$

for

$$
\frac{2}{p}+\frac{1}{q}=\frac{1}{2}, q<\infty
$$

and constants independent of $h$.

The cutoff propagator $S_{l o, 0,0}$ satisfies

$$
\left\|S_{l o, 0,0}\right\|_{L^{2} \rightarrow L_{\alpha h}^{p}(1-m) /(1+m)+\beta} L^{q} \leqslant C h^{-1 / p}
$$

and

$$
\left\|S_{l o, 0,0}\right\|_{L^{2} \rightarrow L_{\alpha h(1-m) /(1+m)}^{p} L^{q}} \leqslant C h^{-(1+\beta) / p},
$$

for $(p, q)$ in the same range and constants independent of $h$.

Remark 4.7. Observe that the parametrix satisfies good Strichartz estimates all the way up to the critical time scale $t \sim h^{(1-m) /(m+1)}$, but we are only able to conclude that the propagator obeys perfect Strichartz estimates on a slightly shorter time scale, or obeys Strichartz estimates with a small loss on the critical time scale. This is an artifact of working in the marginal calculus and trying to make error terms small in $h$.

Proof. We have

$$
\begin{aligned}
v( & t, x) \\
= & T_{h} w(t, x) \\
= & T_{h}(2 \pi)^{-1} \int e^{i \varphi(t, x, \xi)-i y \xi} B(t, x, \xi) \chi_{\star}^{*}\left(y, D_{y}, h\right) w_{0}(y) d y d \xi \\
= & h^{-1 /(m+1)}(2 \pi)^{-1} \int e^{i \varphi\left(h^{(m-1) /(m+1)} t, h^{-1 /(m+1)} x, \xi\right)} \\
& \cdot e^{-i y \xi} B\left(h^{(m-1) /(m+1)} t, h^{-1 /(m+1)} x, \xi\right) \chi_{\star}^{*}\left(y, D_{y}, h\right) w_{0}(y) d y d \xi \\
= & (2 \pi h)^{-1} \int e^{i \varphi_{\star}(t, x, \xi)-i y \xi / h} B_{\star}(t, x, \xi) T_{h} \chi_{\star}^{*}\left(y, D_{y}, h\right) w_{0}(y) d y d \xi,
\end{aligned}
$$

where we use the notation

$$
\varphi_{\star}(t, x, \xi)=\varphi\left(h^{(m-1) /(m+1)} t, h^{-1 /(m+1)} x, h^{-m /(m+1)} \xi\right),
$$

and similarly for $B$. We rewrite this expression as

$$
v_{\star}(t, x)=\int_{y} K_{\star}(t, x, y ; h) \chi_{\star} v_{\star, 0}(y) d y,
$$

where

$$
K_{\star}(t, x, y ; h)=(2 \pi h)^{-1} \int e^{i \varphi_{\star}(t, x, \xi)-i y \xi / h} B_{\star, 0}(t, x, \xi) \tilde{\chi}_{\star}(y, \xi ; h) d \xi,
$$

and

$$
\chi_{\star} v_{\star}, 0(y)=T_{h} \chi_{\star}^{*}\left(y, D_{y}, h\right) w_{0}(y) .
$$


We have already computed the derivative properties of the functions $\varphi$ and $B$ in order to apply the lemma of stationary phase (with $h$ as small parameter). The unique critical point is at

$$
\partial_{\xi}\left(h \varphi_{\star}(t, x, \xi)-y \xi\right)=0,
$$

so the leading asymptotic is

$$
\begin{aligned}
(2 \pi h)^{-1 / 2} & \left|\partial_{\xi}^{2}\left(h \varphi_{\star}(t, x, \xi)-y \xi\right)\right|^{-1 / 2} \\
& =(2 \pi h)^{-1 / 2}\left|h h^{-2 m /(m+1)} \varphi_{\xi \xi}\right|_{\left(h^{(m-1) /(m+1)} t, h^{-1 /(m+1)} x, h^{-m /(m+1)} \xi\right)} \\
& \sim h^{-1 / 2}\left|h h^{-2 m /(m+1)} h^{(m-1) /(m+1)} t\right|^{-1 / 2} \\
& =|h t|^{-1 / 2}
\end{aligned}
$$

as claimed. The Strichartz estimates follow immediately.

We now estimate the difference between the propagator and the parametrix in the $L_{x}^{\infty}$ norm to prove that the actual propagator has the correct dispersion, at least on a slightly shorter time scale. Let $u(t, x)=S_{\mathrm{lo}, 0,0}(t) v_{0}(x)$, so that

$$
\left\{\begin{array}{l}
\left(h D_{t}+P\right)(v-u)=E \\
\left.(v-u)\right|_{t=0}=0
\end{array}\right.
$$

Since the propagator and the parametrix are compactly essentially supported in frequency on scale $h^{-1}$ we have the endpoint Sobolev embeddings:

$$
\sup _{|t| \leqslant \alpha h^{(1-m) /(m+1)+\beta}}\|v-u\|_{L_{x}^{\infty}} \leqslant h^{-1 / 2} \sup _{|t| \leqslant \alpha h^{(1-m) /(m+1)+\beta}}\|v-u\|_{L_{x}^{2}} .
$$

Let the energy $\mathcal{E}(t)=\|v-u\|_{L^{2}}^{2}$, and compute

$$
\begin{aligned}
\mathcal{E}^{\prime} & =2 \operatorname{Re} \frac{i}{h} \int E \overline{(v-u)} d x \\
& \leqslant h^{-1} h^{(1-m) /(m+1)+\beta}\|E\|_{L_{x}^{2}}^{2}+h^{(m-1) /(m+1)+\beta} \mathcal{E},
\end{aligned}
$$

and hence by Gronwall's inequality,

$$
\begin{aligned}
\mathcal{E}(t) & \leqslant C h^{-2 m /(m+1)+\beta}\|E\|_{L_{t}^{2} L_{x}^{2}}^{2} \\
& \leqslant C h^{(1-3 m) /(m+1)+2 \beta}\|E\|_{L_{h^{(1-m) /(m+1)+\beta}}^{\infty} L_{x}^{2}}^{2} \\
& \leqslant C h^{1+2(K+1) \beta}\left\|\chi_{\star}^{*} w_{0}\right\|_{L^{2}}^{2} \\
& \leqslant C h^{2(K+1) \beta}\left\|\chi_{\star}^{*} w_{0}\right\|_{L_{x}^{1}}^{2} .
\end{aligned}
$$

We finally conclude

$$
\begin{aligned}
\sup _{|t| \leqslant \alpha h^{(1-m) /(m+1)+\beta}}\|v-u\|_{L_{x}^{\infty}} & \leqslant C h^{-1 / 2+(K+1) \beta}\left\|\chi_{\star}^{*} w_{0}\right\|_{L_{x}^{1}} \\
& \leqslant C|h t|^{-1 / 2}\left\|\chi_{\star}^{*} w_{0}\right\|_{L_{x}^{1}},
\end{aligned}
$$

provided $|t| \leqslant \alpha h^{(1-m) /(m+1)+\beta}$ and $K$ is sufficiently large that

$$
-\frac{1}{2}+(K+1) \beta \geqslant-\frac{1}{m+1}-\frac{\beta}{2} \text {. }
$$

The Strichartz estimates for $S_{\mathrm{lo}, 0,0}(t)$ follow immediately. 
4.6. The parametrix for $S_{\mathrm{lo}, j,+}^{+}(t)$. The operators $S_{\mathrm{lo}, j,+}^{+}(t)$ are the propagator localized to outgoing frequencies $-a x^{m} \leqslant \xi \leqslant 2 \epsilon$ in the spatial interaction region $\left\{\delta h^{1 /(m+1)} / 2 \leqslant \pm x \leqslant 2 \epsilon\right\}$. We have divided the spatial interaction region into $h$-dependent geometric regions; $S_{\mathrm{lo}, j,+}^{+}(t)$ is localized to

$$
x \in h^{1 /(m+1)} I_{j}:=\left[h^{1 /(m+1)} \delta\left(\omega^{j}-\omega^{j-2}\right), h^{1 /(m+1)} \delta\left(\omega^{j+1}+\omega^{j-1}\right)\right] .
$$

The symbol $\tilde{\chi}\left(\left(\xi+a x^{m}\right) / \gamma x^{m}\right)$ is invariant under the rescaling operation, so after applying the rescaling operators, we are interested in constructing a parametrix in the regions

$$
-a x^{m} \leqslant \xi \leqslant 2 \epsilon h^{-m /(m+1)}, x \in I_{j} .
$$

When the derivative of the effective potential $\widetilde{V}^{\prime}$ is large, singularities propagate away quickly, however not uniformly so. We introduce a loss by constructing $\log (1 / h)$ parametrices, and by eventually restricting our construction to subcritical time scales.

We now compute how long it takes a wave packet to exit the interval $I_{j}$. Write

$$
I_{j}=\left[y_{j}^{-}, y_{j}^{+}\right]:=\left[\delta\left(\omega^{j}-\omega^{j-2}\right), \delta\left(\omega^{j+1}+\omega^{j-1}\right)\right],
$$

and fix an initial point $(y, \eta)$ with $y \in I_{j}, \eta \geqslant-a\left(y_{j}^{+}\right)^{m}$. Then recalling the Hamiltonian system (4.3), we have

$$
x(t) \geqslant y-2 t a\left(y_{j}^{+}\right)^{m} \geqslant \frac{1}{2} y_{j}^{-}
$$

as long as

$$
0 \leqslant t \leqslant \frac{y_{j}^{-}\left(y_{j}^{+}\right)^{-m}}{4 a} .
$$

We have

$$
y_{j}^{+}=y_{j}^{-}\left(\omega+\mathcal{O}\left(\omega^{-1}\right)\right),
$$

so that $x(t) \geqslant y_{j}^{-} / 2$ provided

$$
0 \leqslant t \leqslant \frac{\left(y_{j}^{-}\right)^{1-m}}{4 a \omega^{m}}\left(1+\mathcal{O}\left(\omega^{-1}\right)\right) .
$$

In this case,

$$
-\partial_{x} \widetilde{V} \geqslant\left(y_{j}^{-} / 2\right)^{2 m-1},
$$

which in turn implies

$$
\xi \geqslant-a\left(y_{j}^{+}\right)^{m}+t\left(y_{j}^{-} / 2\right)^{2 m-1} \geqslant b\left(y_{j}^{-}\right)^{m},
$$

provided

$$
t \geqslant 2^{2 m-1}\left(a \omega^{m}\left(1+\mathcal{O}\left(\omega^{-1}\right)\right)+b\right)\left(y_{j}^{-}\right)^{1-m} .
$$

Choosing $a, b>0$ sufficiently small means we can assume $\eta \geqslant b\left(y_{j}^{-}\right)^{m}$ after a time comparable to at most $\left(y_{j}^{-}\right)^{1-m}$. 
We now compute how long it takes to leave $I_{j}$ assuming $y \in I_{j}$ and $\eta \geqslant b\left(y_{j}^{-}\right)^{m}$. We have

$$
\begin{aligned}
x & =y+2 t \eta+\int_{0}^{t}(t-s) B(x(s)) d s \\
& \geqslant y_{j}^{-}+2 t b\left(y_{j}^{-}\right)^{m}+\int_{0}^{t}(t-s) B\left(y_{j}^{-}\right) d s \\
& \geqslant y_{j}^{-}+2 t b\left(y_{j}^{-}\right)^{m}+\frac{1}{2} t^{2}\left(y_{j}^{-}\right)^{2 m-1} \\
& \geqslant y_{j}^{+}
\end{aligned}
$$

provided

$$
t \geqslant\left(y_{j}^{-}\right)^{1-m}\left(-2 b+\sqrt{4 b^{2}+2\left(y_{j}^{+} / y_{j}^{-}-1\right)}\right),
$$

which is again comparable to $\left(y_{j}^{-}\right)^{1-m}$.

We now estimate for $t=\alpha\left(y_{j}^{-}\right)^{1-m}$, for $\alpha>0$ to be determined:

$$
\begin{aligned}
\left|\frac{\partial x}{\partial y}(t)\right| & \leqslant 1+\int_{0}^{t}(t-s)(4 m-2)\left(y_{j}^{+}\right)^{2 m-2} d s\left|\frac{\partial x}{\partial y}(t)\right| \\
& \leqslant 1+(2 m-1) t^{2}\left(y_{j}^{+}\right)^{2 m-2}\left|\frac{\partial x}{\partial y}(t)\right| \\
& \leqslant 1+C_{\omega, m, a, b} \alpha^{2}\left|\frac{\partial x}{\partial y}(t)\right| .
\end{aligned}
$$

Choosing $\alpha>0$ sufficiently small (but independent of $h$ ) shows that

$$
\left|\frac{\partial x}{\partial y}(t)\right| \leqslant C
$$

uniformly for $t$ in this range.

With this estimate in hand, we can compute $\partial x / \partial \eta=2 t(1+\mathcal{O}(t))$ as usual, which results in the following Lemma. In practice, we need to gain some powers of $h$ in our parametrix construction, so we only construct the parametrix up to time $t \sim h^{\epsilon / 2}\left(y_{j}^{-}\right)^{1-m}$ for a small $\epsilon>0$, and then iterate $C h^{-\epsilon}$ times. After time $t \sim h^{-\epsilon / 2}\left(y_{j}^{-}\right)^{1-m}$ then the wavefront set will be outside the interval $I_{j}$. Let us state the following lemma for the short $h$-independent time scale $0 \leqslant t \leqslant \alpha\left(y_{j}^{-}\right)^{1-m}$; we will worry about summing over the $h$-dependent number of time intervals after constructing the amplitude.

Lemma 4.8. There exists $\alpha, a>0$, and $\omega>1$ independent of $h$ and $j$ such that for each $0 \leqslant j \leqslant \mathcal{O}(\log (1 / h))$, there is a phase function $\varphi(t, x, \xi)$ satisfying

$$
\left\{\begin{array}{l}
\varphi_{t}+\varphi_{x}^{2}+\widetilde{V}(x ; h)=0, \\
\varphi(0, x, \eta)=x \eta
\end{array}\right.
$$

for $x \in I_{j},-a\left(y_{j}^{+}\right)^{m} \leqslant \xi \leqslant 2 \epsilon h^{-m /(m+1)}$, and $|t| \leqslant \alpha\left(y_{j}^{-}\right)^{1-m}$.

We further have

$$
\varphi_{\eta \eta} \sim 2 t(1+\mathcal{O}(t))
$$

for $|t| \leqslant \alpha\left(y_{j}^{-}\right)^{1-m}$. 
We now construct the amplitude for the parametrix for the operator $S_{\mathrm{lo}, j,+}^{+}(t)$. This, combined with Lemma 4.8, will be used to compute a dispersion estimate, resulting in a Strichartz estimate. The problem is that, just as in Subsection 4.5, we are working in a marginal calculus, so to construct the amplitude as an asymptotic series, we must restrict the range of $t$ to depend mildly on $h$.

We again appeal to the Frobenius theorem to get a function $\Gamma(t, x)$ (again implicitly depending on the frequency $\xi$ ) satisfying

$$
\left\{\begin{array}{l}
\partial_{t} \Gamma+2 \varphi_{x} \partial_{x} \Gamma=0 \\
\Gamma(0, x)=x
\end{array}\right.
$$

We then construct $B=\sum_{j=0}^{K} B_{j}$ for sufficiently large $K$ to be determined (independent of $h$ ) with

$$
\left\{\begin{array}{l}
B_{0} \equiv 1 \\
B_{j}=-\left.\int_{0}^{t} \varphi_{x x} B_{j-1}\right|_{(s, \Gamma(t-s, x))}+\left.i B_{j-1, x x}\right|_{(s, \Gamma(t-s, x))} .
\end{array}\right.
$$

A tedious induction argument shows that $B_{j}$ satisfies

$$
\left|\partial_{x}^{l} B_{j}\right|=\mathcal{O}\left(\sum_{k=1}^{j}\left|t^{k+j} x^{2 k m-2 j-l}\right|\right) .
$$

Then

$$
w(t, x)=(2 \pi)^{-1} \int e^{i \varphi(t, x, \xi)-i y \xi} B(t, x, \xi) \chi\left(y, D_{y}\right)^{*} w_{0}(y) d y d \xi
$$

solves

$$
\left\{\begin{array}{l}
\widetilde{P} w=\widetilde{E}, \\
w(0, x)=\chi_{\star}\left(x, D_{x}\right)^{*} w_{0}(x),
\end{array}\right.
$$

where

$$
\chi_{\star}=T_{h}^{-1} \psi\left( \pm \omega^{j} x / h^{1 /(m+1)}\right)\left(1-\mathbb{1}_{\left\{ \pm h D_{x} \geqslant 1-V(x)\right\}}\left(1-\chi_{\epsilon^{2}}((P-1))\right)\right) \chi_{\epsilon}(x) T_{h}
$$

is the appropriate microlocal cutoff, and the equation is understood to make sense for $|t| \leqslant \alpha\left(y_{j}^{-}\right)^{1-m}$. Here, the error $\widetilde{E}$ is given by

$$
\widetilde{E}=(2 \pi)^{-1} \int e^{i \varphi(t, x, \xi)-i y \xi}\left(-\partial_{x}^{2} B_{K}-i \varphi_{x x} B_{K}\right) \chi^{*}\left(y, D_{y}\right) w_{0}(y) d y d \xi
$$

That is, $\widetilde{E}$ is an oscillatory integral operator with the same phase function as $w$. Having computed the symbol of the error term $\widetilde{E}$ to be $-\partial_{x}^{2} B_{K}-i \varphi_{x x} B_{K}$, in the rescaled coordinates we have for $|t| \leqslant h^{\beta / 2}|x|^{1-m}$,

$$
\begin{aligned}
-\partial_{x}^{2} B_{K}-i \varphi_{x x} B_{K} & =\mathcal{O}\left(\sum_{l=1}^{K+1}|t|^{l+K}|x|^{2 m l-2 K-2}\right) \\
& =\mathcal{O}\left(\sum_{l=1}^{K+1} h^{(l+K) \beta / 2}|x|^{(m+1)(l-K)-2}\right) \\
& =\mathcal{O}\left(h^{(1+K) \beta / 2}|x|^{m-1}\right)
\end{aligned}
$$

in the worst case when $l=K+1$. Now since $|x| \leqslant h^{-1 /(m+1)}$, this error term is of order $\mathcal{O}\left(h^{(1+K) \beta / 2+(1-m) /(1+m)}\right)$, which is small as $K$ gets large. 
If we now take $v=T_{h} w$, we see

$$
\begin{aligned}
P v & =T_{h} T_{h}^{-1} P T_{h} w \\
& =h^{2 m /(m+1)} T_{h} \widetilde{P} w \\
& =E,
\end{aligned}
$$

with initial conditions

$$
v(0, x)=T_{h} w(0, x),
$$

and where

$$
E=h^{2 m /(m+1)} T_{h} \widetilde{E} .
$$

A similar computation to Subsection 4.5 shows

$$
\sup _{0 \leqslant t \leqslant \alpha h^{\beta / 2}\left|y_{j}^{-}\right|^{1-m}}\|E\|_{L^{2}} \leqslant C h^{2 m /(m+1)} h^{\beta(1+K) / 2+(1-m) /(1+m)}\left\|\chi_{\star}^{*} v_{0}\right\|_{L^{2}} .
$$

The following lemma contains the dispersion and Strichartz estimates for the operators $S_{\mathrm{lo}, j,+}^{+}(t)$.

Lemma 4.9. The parametrix $v(t, x)$ satisfies the dispersion estimate

$$
\left\|\chi_{\star} v\right\|_{L^{\infty}} \leqslant C(h t)^{-1 / 2}\left\|\tilde{\chi} v_{0}\right\|_{L^{1}},
$$

where $0<t \leqslant \alpha h^{\beta / 2}\left|y_{j}^{-}\right|^{1-m}$, as well as the corresponding Strichartz estimate

$$
\|v\|_{L_{\alpha h^{\beta / 2}\left|y_{j}^{-}\right| 1-m}^{p} L^{q}} \leqslant C h^{-1 / p}\left\|\chi v_{0}\right\|_{L^{2}},
$$

for

and constants independent of $h$.

$$
\frac{2}{p}+\frac{1}{q}=\frac{1}{2}, q<\infty
$$

The cutoff propagator $S_{l o, j,+}^{+}$satisfies

$$
\left\|S_{l o, j,+}^{+}\right\|_{L^{2} \rightarrow L_{\alpha h^{\beta / 2}\left|y_{j}^{-}\right|^{1-m}}^{p} L^{q}} \leqslant C h^{-1 / p}
$$

and

$$
\left\|S_{l o, j,+}^{+}\right\|_{L^{2} \rightarrow L_{\alpha h(1-m) /(1+m)}^{p} L^{q}} \leqslant C h^{-(1+\beta) / p},
$$

for $(p, q)$ in the same range and constants independent of $h$.

The proof is exactly the same as the proof of Lemma 4.6 with the exception of the different time interval. If we sum over $h^{-\beta}$ intervals of length $h^{\beta / 2}\left|y_{j}^{-}\right|^{1-m}$ results in an interval of length $h^{-\beta / 2}\left|y_{j}^{-}\right|^{1-m}$. According to Lemma 4.8 (combined with the Egorov theorem in the $h^{-1 / 2+\beta}$ calculus), after this time the parametrix and the error are both $\mathcal{O}\left(h^{\infty}\right)$.

4.7. Proof of Proposition [3.2. In this subsection, we see how to use the computed Strichartz estimates plus the local smoothing from CW11 to prove Proposition 3.2

From the semiclassical Strichartz estimates, if we let $v(t, x)=v_{l k}(t h, x)$ as in Proposition 3.2 and rescale appropriately, we get

$$
\left\|\chi v_{l k}\right\|_{L_{T}^{2 n} L^{2^{\star}}} \leqslant C_{\beta}\left\|\langle k\rangle^{\beta} v_{l k}^{0}\right\|_{L^{2}},
$$

for $T \leqslant \epsilon k^{-2 /(m+1)}$, and where $\chi \in \mathcal{C}_{c}^{\infty}$ is any smooth, compactly supported function. Recall that according to Lemmas 4.1 , we already have perfect Strichartz 
estimates for $(1-\chi) v_{l k}$ if $\chi \equiv 1$ near $x=0$. Further, by Lemma 4.2, we have perfect Strichartz estimates for large frequencies and small $x$ : if $\psi(\xi) \equiv 1$ near 0 , $\chi\left(1-\psi\left(-h^{2} \Delta\right)\right) v_{l k}$ obeys perfect Strichartz estimates.

Let $\chi$ and $\psi$ be such cutoffs. In order to estimate $\chi \psi v_{l k}$, we employ a duality trick (see BGH10]) together with the local smoothing estimates from CW11. Let $\varphi(s) \in \mathcal{C}_{c}^{\infty}$ be a compactly supported function such that

$$
\sum_{j=0}^{k^{2 /(m+1)}} \varphi\left(k^{2 /(m+1)} t-j\right) \equiv 1, \quad 0 \leqslant t \leqslant \epsilon .
$$

Set $U_{j}=\varphi\left(k^{2 /(m+1)} t-j\right) \chi \psi v_{j k}$. We have

$$
\left(D_{t}+P_{k}\right) U_{j}=W_{j}^{\prime}+W_{j}^{\prime \prime}
$$

where

$$
W_{j}^{\prime}=i k^{2 /(m+1)} \varphi^{\prime}\left(k^{2 /(m+1)} t-j\right) \chi \psi v_{j k},
$$

and

$$
W_{j}^{\prime \prime}=\varphi\left(\chi^{\prime \prime}+2 \chi^{\prime} \partial_{x}\right) \psi v_{l k}
$$

The important thing to observe is that $W_{j}^{\prime \prime}$ is supported away from $x=0$, so the standard 1/2 derivative local smoothing estimates hold (see Theorem 2). Let $\chi_{1} \in \mathcal{C}_{c}^{\infty}$ satisfy $\chi_{1} \equiv 1$ on $\operatorname{supp} \chi$, and $\chi_{2} \in \mathcal{C}_{c}^{\infty}$ satisfy $\chi_{2} \equiv 1$ on supp $\chi^{\prime}, \operatorname{supp} \chi_{2}$ away from $x=0$. We have $\chi_{1} U_{j}=U_{j}, \chi_{1} W_{j}^{\prime}=W_{j}^{\prime}$, and $\chi_{2} W_{j}^{\prime \prime}=W_{j}^{\prime \prime}$. Using the Duhamel formula, set

$$
U_{j}^{\prime}=\chi_{1} \int_{(j-1) \epsilon k^{-2 /(m+1)}}^{t} e^{-i(t-s) P_{k}} \chi_{1} W_{j}^{\prime}(s) d s,
$$

and

$$
U_{j}^{\prime \prime}=\chi_{1} \int_{(j-1) \epsilon k^{-2 /(m+1)}}^{t} e^{-i(t-s) P_{k}} \chi_{2} W_{j}^{\prime \prime}(s) d s,
$$

so that $U_{j}^{\prime}+U_{j}^{\prime \prime}=U_{j}$. By the Christ-Kiselev lemma CK01, it suffices to consider

$$
\bar{U}_{j}^{\prime}=\chi_{1} \int_{(j-1) \epsilon k^{-2 /(m+1)}}^{(j+1) \epsilon k^{-2 /(m+1)}} e^{-i(t-s) P_{k}} \chi_{1} W_{j}^{\prime}(s) d s,
$$

and similarly for $W_{j}^{\prime \prime}$. Let $I=\left[(j-1) \epsilon k^{-2 /(m+1)},(j+1) \epsilon k^{-2 /(m+1)}\right]$ be the time interval in the integral above. We apply the Strichartz estimates to get

$$
\left\|\bar{U}_{j}^{\prime}\right\|_{L_{I}^{2 n} L^{2^{\star}}} \leqslant C k^{\beta}\left\|\int_{(j-1) \epsilon k^{-2 /(m+1)}}^{(j+1) \epsilon k^{-2 /(m+1)}} e^{i s P_{k}} \chi_{1} W_{j}^{\prime}(s) d s\right\|_{L^{2}},
$$

and similarly for $W_{j}^{\prime \prime}$. The dual estimates to Theorem 2 then yield

$$
\left\|\bar{U}_{j}^{\prime}\right\|_{L_{I}^{2 n} L^{2^{\star}}} \leqslant C k^{\beta-1 /(m+1)}\left\|W_{j}^{\prime}\right\|_{L^{2} L^{2}}
$$

and (again because $\chi_{2}$ is supported away from $x=0$ )

$$
\left\|\bar{U}_{j}^{\prime \prime}\right\|_{L_{I}^{2 n} L^{2 \star}} \leqslant C k^{\beta-1 / 2}\left\|W_{j}^{\prime \prime}\right\|_{L^{2} L^{2}} .
$$


By the Christ-Kiselev lemma CK01, the same estimates hold for $U_{j}^{\prime}$ and $U_{j}^{\prime \prime}$. Squaring and summing in $j$, using $\ell^{2} \subset \ell^{2 n}$, yields

$$
\begin{aligned}
\left\|v_{l k}\right\|_{L_{\epsilon}^{2 n} L^{2^{\star}}}^{2} & \leqslant C \sum_{j=0}^{k^{2 /(m+1)}}\left(\left\|U_{j}^{\prime}\right\|_{L_{\epsilon}^{2 n} L^{2^{\star}}}^{2}+\left\|U_{j}^{\prime \prime}\right\|_{L_{\epsilon}^{2 n} L^{2^{\star}}}^{2}\right) \\
& \leqslant C \sum_{j=0}^{k^{2 /(m+1)}}\left(k^{2 \beta-2 /(m+1)}\left\|W_{j}^{\prime}\right\|_{L^{2} L^{2}}^{2}+k^{2 \beta-1}\left\|W_{j}^{\prime \prime}\right\|_{L^{2} L^{2}}^{2}\right) \\
& \leqslant C\left(k^{2 \beta+2 /(m+1)}\left\|\chi v_{j k}\right\|_{L_{\epsilon}^{2} L^{2}}^{2}+k^{2 \beta-1}\left\|\chi_{2}\left\langle D_{x}\right\rangle v_{l k}\right\|_{L_{\epsilon}^{2} L^{2}}^{2}\right) \\
& \leqslant C k^{2 \beta}\left\|v_{l k}^{0}\right\|_{L^{2}}^{2} .
\end{aligned}
$$

This proves Proposition 3.2 .

\section{Quasimodes}

In this section we construct quasimodes for the model operator near $(0,0)$ in the transversal phase space, and then use these quasimodes to show the Strichartz estimates are near-sharp, in the sense described in Corollary 3.3 .

Consider the model operator

$$
P=-h^{2} \partial_{x}^{2}-m^{-1} x^{2 m}
$$

locally near $x=0$. We will construct quasimodes which are localized very close to $x=0$, so this should be a decent approximation. It is well-known (see [CW11]) that the operator

$$
\tilde{Q}=-\partial_{x}^{2}+x^{2 m}
$$

has a unique ground state $\tilde{Q} v_{0}=\lambda_{0} v_{0}$, with $\lambda_{0}>0$, and $v_{0}$ is a Schwartz class function. Then, by rescaling, we find the function $v(x)=v_{0}\left(x h^{-1 /(m+1)}\right)$ is an un-normalized eigenfunction for the equation

$$
\left(-h^{2} \partial_{x}^{2}+x^{2 m}\right) v=h^{2 m /(m+1)} \lambda_{0} v .
$$

Complex scaling then suggests there are resonances with imaginary part $c_{0} h^{2 m /(m+1)}$. We use a complex WKB approximation to get an explicit formula for a localized approximate resonant state, however, as we shall see, it is not a very good approximation. Nevertheless, since we will eventually be averaging in time, it is sufficient for our applications.

Let $E_{0}=(\alpha+i \mu) h^{2 m /(m+1)}, \alpha, \mu>0$ independent of $h$. Let the phase function

$$
\varphi(x)=\int_{0}^{x}\left(E+m^{-1} y^{2 m}\right)^{1 / 2} d y,
$$

where the branch of the square root is chosen to have positive imaginary part. Let

$$
u(x)=\left(\varphi^{\prime}\right)^{-1 / 2} e^{i \varphi / h},
$$

so that

$$
(h D)^{2} u=\left(\varphi^{\prime}\right)^{2} u+f u,
$$

where

$$
\begin{aligned}
f & =\left(\varphi^{\prime}\right)^{1 / 2}(h D)^{2}\left(\varphi^{\prime}\right)^{-1 / 2} \\
& =-h^{2}\left(\frac{3}{4}\left(\varphi^{\prime}\right)^{-2}\left(\varphi^{\prime \prime}\right)^{2}-\frac{1}{2}\left(\varphi^{\prime}\right)^{-1} \varphi^{\prime \prime \prime}\right) .
\end{aligned}
$$


Lemma 5.1. The phase function $\varphi$ satisfies the following properties:

(i): There exists $C>0$ independent of $h$ such that

$$
|\operatorname{Im} \varphi| \leqslant C\left\{\begin{array}{l}
h\left(1+\log \left(x / h^{1 / 2}\right)\right), \quad m=1, \\
h, \quad m \geqslant 2 .
\end{array}\right.
$$

In particular, if $|x| \leqslant C h^{1 /(m+1)},|\operatorname{Im} \varphi| \leqslant C^{\prime}$ for some $C^{\prime}>0$ independent of $h$.

(ii): There exists $C>0$ independent of $h$ such that

$$
C^{-1} \sqrt{h^{2 m /(m+1)}+x^{2 m}} \leqslant\left|\varphi^{\prime}(x)\right| \leqslant C \sqrt{h^{2 m /(m+1)}+x^{2 m}}
$$

(iii):

$$
\left\{\begin{array}{l}
\varphi^{\prime}=\left(E+m^{-1} x^{2 m}\right)^{1 / 2} \\
\varphi^{\prime \prime}=x^{2 m-1}\left(\varphi^{\prime}\right)^{-1} \\
\varphi^{\prime \prime \prime}=\left(\left(1-m^{-1}\right) x^{4 m-2}+E(2 m-1) x^{2 m-2}\right)\left(\varphi^{\prime}\right)^{-3}
\end{array}\right.
$$

In particular,

$$
f=-h^{2} x^{2 m-2}\left(\left(\frac{1}{4}+\frac{1}{2 m}\right) x^{2 m}-\left(m-\frac{1}{2}\right) E\right)\left(\varphi^{\prime}\right)^{-4} .
$$

Proof. For (i) we write $\varphi^{\prime}=s+i t$ for $s$ and $t$ real valued, and then

$$
E+m^{-1} x^{2 m}=s^{2}-t^{2}+2 i s t .
$$

Hence

$$
s^{2} \geqslant s^{2}-t^{2}=\alpha h^{2 m /(m+1)}+m^{-1} x^{2 m}
$$

so that

$$
t=\frac{\mu h^{2 m /(m+1)}}{2 s} \leqslant \frac{\mu h^{2 m /(m+1)}}{2 \sqrt{h^{2 m /(m+1)} \alpha+m^{-1} x^{2 m}}} .
$$

Then

$$
\begin{aligned}
|\operatorname{Im} \varphi(x)| & \leqslant \int_{0}^{|x|} \varphi^{\prime}(y) d y \\
& \leqslant C \int_{0}^{h^{1 /(m+1)}} h^{m /(m+1)} d y+C \int_{h^{1 /(m+1)}}^{x} h^{2 m /(m+1)} y^{-m} d y \\
& = \begin{cases}\mathcal{O}\left(h\left(1+\log \left(x / h^{1 / 2}\right)\right)\right), & m=1, \\
\mathcal{O}(h), & m>1 .\end{cases}
\end{aligned}
$$

Parts (ii) and (iii) are simple computations.

In light of this lemma, $|u(x)|$ is comparable to $\left|\varphi^{\prime}\right|^{-1 / 2}$, provided $|x| \leqslant C h^{1 / 2}$ when $m=1$. We are only interested in sharply localized quasimodes and in the case $m \geqslant 2$, so let $\gamma=h^{1 /(m+1)}$, choose $\chi(s) \in \mathcal{C}_{c}^{\infty}(\mathbb{R})$ such that $\chi \equiv 1$ for $|s| \leqslant 1$ and $\operatorname{supp} \chi \subset[-2,2]$. Let

$$
\tilde{u}(x)=\chi(x / \gamma) u(x)
$$


and compute for $q \geqslant 2$ :

$$
\begin{aligned}
\|\tilde{u}\|_{L^{q}}^{q} & =\int_{|x| \leqslant 2 \gamma} \chi(x / \gamma)^{q}|u|^{q} d x \\
& \sim \int_{|x| \leqslant 2 \gamma} \chi(x / \gamma)^{q}\left|\varphi^{\prime}\right|^{-q / 2} d x \\
& \sim h^{1 /(m+1)} h^{-q m / 2(m+1)} \\
& \sim h^{(2-q m) / 2(1+m)} .
\end{aligned}
$$

In particular,

$$
\|\tilde{u}\|_{L^{2}} \sim h^{(1-m) / 2(1+m)}
$$

and so

$$
\|\tilde{u}\|_{L^{q}} \sim h^{(2 / q-1) / 2(m+1)}\|\tilde{u}\|_{L^{2}} .
$$

Further, $\tilde{u}$ satisfies the following equation:

$$
\begin{aligned}
(h D)^{2} \tilde{u} & =\chi(x / \gamma)(h D)^{2} u+\left[(h D)^{2}, \chi(x / \gamma)\right] u \\
& =\left(\varphi^{\prime}\right)^{2} \tilde{u}+f \tilde{u}+\left[(h D)^{2}, \chi(x / \gamma)\right] u \\
& =\left(\varphi^{\prime}\right)^{2} \tilde{u}+R
\end{aligned}
$$

where

$$
R=f \tilde{u}+\left[(h D)^{2}, \chi(x / \gamma)\right] u .
$$

Lemma 5.2. The remainder $R$ satisfies

$$
\|R\|_{L^{2}}=\mathcal{O}\left(h^{2 m /(m+1)}\right)\|\tilde{u}\|_{L^{2}} .
$$

Proof. We have already computed the function $f$, which is readily seen to satisfy

$$
\|f\|_{L^{\infty}(\operatorname{supp}(\tilde{u}))}=\mathcal{O}\left(h^{2 m /(m+1)}\right),
$$

since $\operatorname{supp}(\tilde{u}) \subset\left\{|x| \leqslant 2 h^{1 /(m+1)}\right\}$.

On the other hand, since $\|\tilde{u}\|_{L^{2}} \sim h^{(1-m) / 2(1+m)}$, we need only show that

$$
\left\|\left[(h D)^{2}, \chi(x / \gamma)\right] u\right\|_{L^{2}} \leqslant C h^{(3 m+1) / 2(m+1)} .
$$

We compute:

$$
\begin{aligned}
{\left[(h D)^{2}, \chi(x / \gamma)\right] u } & =-h^{2} \gamma^{-2} \chi^{\prime \prime} u+2 \frac{h}{i} \gamma^{-1} \chi^{\prime} h D u \\
& =-h^{2} \gamma^{-2} \chi^{\prime \prime} u+2 \frac{h}{i} \gamma^{-1} \chi^{\prime}\left(-\frac{h}{2 i} \frac{\varphi^{\prime \prime}}{\varphi^{\prime}}+\varphi^{\prime}\right) u \\
& =-h^{2} \gamma^{-2} \chi^{\prime \prime} u+2 \frac{h}{i} \gamma^{-1} \chi^{\prime}\left(-\frac{h}{2 i} \frac{x^{2 m-1}}{\left(\varphi^{\prime}\right)^{2}}+\varphi^{\prime}\right) u .
\end{aligned}
$$

The first term is estimated:

$$
\left\|h^{2} \gamma^{-2} \chi^{\prime \prime} u\right\|_{L^{2}}=\mathcal{O}\left(h^{2 m /(m+1)}\right)\|u\|_{L^{2}(\operatorname{supp}(\tilde{u}))}=\mathcal{O}\left(h^{(3 m+1) / 2(m+1)}\right) .
$$


Similarly, the remaining two terms are estimated:

$$
\begin{aligned}
\left\|2 \frac{h}{i} \gamma^{-1} \chi^{\prime}\left(-\frac{h}{2 i} \frac{x^{2 m-1}}{\left(\varphi^{\prime}\right)^{2}}+\varphi^{\prime}\right) u\right\|_{L^{2}} \\
=\mathcal{O}\left(h^{m /(m+1)} h^{1} h^{(2 m-1) /(m+1)} h^{-2 m /(m+1)}\right)\|u\|_{L^{2}(\operatorname{supp}(\tilde{u}))} \\
\quad+\mathcal{O}\left(h^{m /(m+1)} h^{2 m /(m+1)}\right)\|u\|_{L^{2}(\operatorname{supp}(\tilde{u}))} \\
=\mathcal{O}\left(h^{(3 m+1) / 2(m+1)}\right) .
\end{aligned}
$$

5.1. Saturation of Strichartz estimates. In this subsection, we study Strichartz estimates for the separated Schrödinger equation given the specific choice of initial conditions in the form of quasimodes.

Now it is well known that for any $k$, there exists a spherical harmonic $v_{k}$ of order $k$ which saturates Sogge's bounds (Theorem 31):

$$
-\Delta_{\mathbb{S}^{d}} v_{k}=(k)(k+d-1) v_{k}, \quad\left\|v_{k}\right\|_{L^{2(d+1) /(d-1)}} \sim k^{(d-1) / 2(d+1)}\left\|v_{k}\right\|_{L^{2}} .
$$

Let $\lambda_{k}=k(k+n-2), k \gg 1, h=\lambda_{k}^{-1 / 2}$, let $\tilde{u}$ be the associated transversal quasimode constructed in the previous section, and let

$$
\varphi_{0}(x, \theta)=v_{k}(\theta) \tilde{u}(x) .
$$

Let $\varphi(t, x, \theta)=e^{i t \tau} \varphi_{0}$ for some $\tau \in \mathbb{C}$ to be determined. Since the support of $\tilde{u}$ is very small, contained in $\left\{|x| \leqslant h^{1 /(m+1)} / \kappa\right\}$, we have

$$
A^{-2}=\left(1+x^{2 m}\right)^{-1 / m}=1-\frac{1}{m} x^{2 m}+\mathcal{O}\left(h^{4 m /(m+1)}\right)
$$

on $\operatorname{supp} \tilde{u}$. Then

$$
\begin{aligned}
\left(D_{t}+\widetilde{\Delta}\right) \varphi & =P_{k} \varphi \\
& =\left(\tau-D_{x}^{2}-A^{-2} \lambda_{k}-V_{1}(x)\right) \varphi \\
& =\lambda_{k} e^{i t \tau} e^{i k \theta}\left[\left(\tau \lambda_{l}^{-1}-\left(\lambda_{k}^{-1} D_{x}^{2}+1-\frac{1}{m} x^{2 m}\right)\right) \tilde{u}+\mathcal{O}\left(k^{-2}\right) \tilde{u}\right] \\
& =\lambda_{k} e^{i t \tau} e^{i k \theta}\left[\left(\tau \lambda_{k}^{-1}-1-E_{0}\right) \tilde{u}+R+\mathcal{O}\left(k^{-2}\right) \tilde{u}\right],
\end{aligned}
$$

where $R$ satisfies the remainder estimate (5.1). Set

$$
\tau=\lambda_{k}\left(1+E_{0}\right)=\lambda_{k}\left(1+\alpha k^{-2 m /(m+1)}\right)+i \mu k^{2 /(m+1)}\left(1+\mathcal{O}\left(k^{-1}\right)\right), \quad \alpha, \mu>0
$$

so that we have

$$
\left\{\begin{array}{l}
\left(D_{t}+\widetilde{\Delta}\right) \varphi=\tilde{R}, \\
\varphi(0, x, \theta)=\varphi_{0}
\end{array}\right.
$$

with

$$
\tilde{R}=\lambda_{k} e^{i t \tau} v_{k}\left(R(x, k)+\mathcal{O}\left(k^{-2}\right) \tilde{u}\right) .
$$


We compute the endpoint Strichartz estimate on an arbitrary time interval $[0, T]$, with $p=2, q=2^{\star}=2 n /(n-2)$ for $n \geqslant 3$ :

$$
\begin{aligned}
\|\varphi\|_{L^{2}([0, T]) L^{q}}^{2} & =\int_{0}^{T}\left\|e^{i t \tau} \varphi_{0}\right\|_{L^{q}}^{2} d t \\
& =\int_{0}^{T} e^{-2 t \operatorname{Im} \tau}\left\|\varphi_{0}\right\|_{L^{q}}^{2} d t \\
& =\frac{1-e^{-2 T \operatorname{Im} \tau}}{2 \operatorname{Im} \tau}\left\|\varphi_{0}\right\|_{L^{q}}^{2} \\
& =\frac{1-e^{-2 T \operatorname{Im} \tau}}{2 \operatorname{Im} \tau}\left\|\varphi_{0}\right\|_{L^{q}}^{2} \\
& \sim \frac{1-e^{-2 T \operatorname{Im} \tau}}{2 \operatorname{Im} \tau} k^{(1-2 / q) /(m+1)} k^{(n-2) / n}\|\tilde{u}\|_{L^{2}(\mathbb{R})}^{2}\left\|v_{k}\right\|_{L^{2}\left(\mathbb{S}^{n-1}\right)}^{2} \\
& \sim k^{2 \eta(m, n)}\left\|\varphi_{0}\right\|_{L^{2}}^{2} \\
& \sim\left\|\left(-\Delta_{\mathbb{S}^{n-1}}\right)^{\eta(m, n)} \varphi_{0}\right\|_{L^{2}}^{2}
\end{aligned}
$$

where

$$
\eta(m, n)=\frac{1}{2(m+1)}\left(m\left(1-\frac{2}{n}\right)-1\right) .
$$

Now let $L(t)$ be the unitary Schrödinger propagator:

$$
\left\{\begin{array}{l}
\left(D_{t}+\widetilde{\Delta}\right) L=0, \\
L(0)=\text { id },
\end{array}\right.
$$

and write using Duhamel's formula:

$$
\varphi(t)=L(t) \varphi_{0}+i \int_{0}^{t} L(t) L^{*}(s) \tilde{R}(s) d s=: \varphi_{\mathrm{h}}+\varphi_{\mathrm{ih}},
$$

where $\varphi_{\mathrm{h}}$ and $\varphi_{\mathrm{ih}}$ are the homogeneous and inhomogeneous parts respectively. We want a lower bound on the homogeneous Strichartz estimates, for which we need an upper bound on the inhomogeneous Strichartz estimates.

Let us now assume for the purposes of contradiction that a better Strichartz estimate than that in Corollary 3.3 holds for all $\beta>0$. That is, we assume for each $\beta>0$, there exists $C_{\beta}$ such that

$$
\left\|L(t) u_{0}\right\|_{L^{2}([0, T]) L^{2^{\star}}} \leqslant C_{\beta}\left\|\left\langle-\Delta_{\mathbb{S} n-1}\right\rangle^{r+\beta} u_{0}\right\|_{L^{2}},
$$

for some $r<\eta(m, n) / 2$.

In dimension $n=2$, we take as usual $p>2,2 \leqslant q<\infty$, and we immediately arrive at a contradiction to the scale-invariant case.

For dimension $n \geqslant 3$, we take $\beta>0$ sufficiently small that $r+\beta<\eta(m, n) / 2$, and we then have the complementary inhomogeneous Strichartz estimate: if $v$ solves

$$
\left\{\begin{array}{l}
\left(D_{t}+\widetilde{\Delta}\right) v=F \\
v(0)=0
\end{array}\right.
$$

then

$$
\|v\|_{L^{2}([0, T]) L^{2^{\star}}} \leqslant C\left\|\left\langle-\Delta_{\mathbb{S}^{n-1}}\right\rangle^{r+\beta} F\right\|_{L^{1}([0, T]) L^{2}} .
$$


For the inhomogeneous part corresponding to our quasimode initial data, we have $F=\tilde{R}$, with $\tilde{R}$ computed in (5.2). Then

$$
\begin{aligned}
& \left\|\varphi_{\mathrm{ih}}\right\|_{L^{2}([0, T]) L^{2^{\star}}} \\
& \leqslant C T^{1 / 2}\|\tilde{R}\|_{L^{2}([0, T]) L^{2}} \\
& \leqslant C k^{2} T^{1 / 2}\left(\int_{0}^{T} e^{-2 t \operatorname{Im} \tau}\left\|\left\langle-\Delta_{\mathbb{S}^{n-1}}\right\rangle^{r+\beta} v_{k}\left(R(x, k)+\mathcal{O}\left(k^{-2}\right) \tilde{u}\right)\right\|_{L^{2}}^{2} d t\right)^{1 / 2} \\
& \leqslant C k^{2} k^{-2 m /(m+1)}\left(\frac{1-e^{-2 T \operatorname{Im} \tau}}{2 \operatorname{Im} \tau}\right)^{1 / 2}\left\|\left\langle-\Delta_{\mathbb{S}^{n-1}}\right\rangle^{r+\beta} \varphi_{0}\right\|_{L^{2}} .
\end{aligned}
$$

Recalling that $\operatorname{Im} \tau \sim k^{-2 /(m+1)}$, if $T=\epsilon^{2} k^{-2 /(m+1)}$, we have

$$
\left\|\varphi_{\mathrm{h}}\right\|_{L^{2}([0, T]) L^{2^{\star}}} \leqslant C \epsilon\left\|\left\langle-\Delta_{\mathbb{S}^{n-1}}\right\rangle^{r+\beta} \varphi_{0}\right\|_{L^{2}} .
$$

Now, if $\epsilon>0$ is sufficiently small, but independent of $k$, we have

$$
1 \geqslant 1-e^{-2 T \operatorname{Im} \tau} \geqslant c_{0},
$$

for some $c_{0}>0$, so that for this choice of $T$, we still have the estimate (5.3). Combining (5.3) with (5.4) we have

$$
\begin{aligned}
C\left\|\left\langle-\Delta_{\mathbb{S}^{n-1}}\right\rangle^{r+\beta} \varphi_{0}\right\|_{L^{2}} & \geqslant\left\|L(t) \varphi_{0}\right\|_{L^{2}([0, T]) L^{2^{\star}}} \\
& \geqslant\|\varphi(t)\|_{L^{2}([0, T]) L^{2^{\star}}}-\left\|\varphi_{\mathrm{ih}}\right\|_{L^{2}([0, T]) L^{2^{\star}}} \\
& \geqslant C^{-1}\left\|\left\langle-\Delta_{\mathbb{S}^{n-1}}\right\rangle^{\eta(m, n) / 2} \varphi_{0}\right\|_{L^{2}},
\end{aligned}
$$

for some constant $C>0$ independent of $k$. But this is a contradiction, since $r+\beta<\eta(m, n) / 2$. This proves the near-sharpness of Corollary 3.3 .

\section{REFERENCES}

[Ana08] Nalini Anantharaman. Entropy and the localization of eigenfunctions. Ann. of Math. (2), 168(2):435-475, 2008.

[BGH10] Nicolas Burq, Colin Guillarmou, and Andrew Hassell. Strichartz estimates without loss on manifolds with hyperbolic trapped geodesics. Geom. Funct. Anal., 20(3):627656, 2010.

[BGT04] Nicolas Burq, Patrick Gérard, and Nikolay Tzvetkov. Strichartz inequalities and the nonlinear Schrödinger equation on compact manifolds. Amer. J. Math., 126(3):569605, 2004.

[BH08] Jean-François Bony and Dietrich Häfner. Decay and non-decay of the local energy for the wave equation on the de Sitter-Schwarzschild metric. Comm. Math. Phys., 282(3):697-719, 2008.

[BS03] P. Blue and A. Soffer. Semilinear wave equations on the Schwarzschild manifold. I. Local decay estimates. Adv. Differential Equations, 8(5):595-614, 2003.

[BT08] Jean-Marc Bouclet and Nikolay Tzvetkov. On global Strichartz estimates for nontrapping metrics. J. Funct. Anal., 254(6):1661-1682, 2008.

[Bur04] N. Burq. Smoothing effect for Schrödinger boundary value problems. Duke Math. J., 123(2):403-427, 2004.

[BZ04] Nicolas Burq and Maciej Zworski. Geometric control in the presence of a black box. J. Amer. Math. Soc., 17(2):443-471 (electronic), 2004.

[Chr07] Hans Christianson. Semiclassical non-concentration near hyperbolic orbits. J. Funct. Anal., 246(2):145-195, 2007.

[Chr08a] Hans Christianson. Cutoff resolvent estimates and the semilinear Schrödinger equation. Proc. Amer. Math. Soc., 136:3513-3520, 2008.

[Chr08b] Hans Christianson. Dispersive estimates for manifolds with one trapped orbit. Comm. Partial Differential Equations, 33:1147-1174, 2008. 
[Chr09] Hans Christianson. Applications of cutoff resolvent estimates to the wave equation. Math. Res. Lett., 16(4):577-590, 2009.

[Chr11] Hans Christianson. Quantum monodromy and nonconcentration near a closed semihyperbolic orbit. Trans. Amer. Math. Soc., 363(7):3373-3438, 2011.

[CK01] Michael Christ and Alexander Kiselev. Maximal functions associated to filtrations. $J$. Funct. Anal., 179(2):409-425, 2001.

[CW11] Hans Christianson and Jared Wunsch. Local smoothing for the schrödinger equation with a prescribed loss. preprint, 2011.

[Dat09] Kiril Datchev. Local smoothing for scattering manifolds with hyperbolic trapped sets. Comm. Math. Phys., 286(3):837-850, 2009.

[DR09] Mihalis Dafermos and Igor Rodnianski. The red-shift effect and radiation decay on black hole spacetimes. Comm. Pure Appl. Math., 62(7):859-919, 2009.

[KT98] Markus Keel and Terence Tao. Endpoint Strichartz estimates. Amer. J. Math., 120(5):955-980, 1998.

[LMar] Parul Laul and Jason Metcalfe. Localized energy estimates for wave equations on high dimensional Schwarzschild space-times. Proc. Amer. Math. Soc., to appear.

[Luk10] Jonathan Luk. Improved decay for solutions to the linear wave equation on a Schwarzschild black hole. Ann. Henri Poincaré, 11(5):805-880, 2010.

[MMTT10] Jeremy Marzuola, Jason Metcalfe, Daniel Tataru, and Mihai Tohaneanu. Strichartz estimates on Schwarzschild black hole backgrounds. Comm. Math. Phys., 293(1):3783, 2010.

[NZ09] Stéphane Nonnenmacher and Maciej Zworski. Quantum decay rates in chaotic scattering. Acta Math., 203(2):149-233, 2009.

[Sog86] Christopher D. Sogge. Oscillatory integrals and spherical harmonics. Duke Math. J., 53(1):43-65, 1986.

[TT11] Daniel Tataru and Mihai Tohaneanu. A local energy estimate on Kerr black hole backgrounds. Int. Math. Res. Not. IMRN, 2011(2):248-292, 2011.

[WZ10] Jared Wunsch and Maciej Zworski. Resolvent estimates for normally hyperbolic trapped sets. Ann. Henri Poincaré, to appear, 2010.

E-mail address: hans@math.unc.edu

Department of Mathematics, UnC-Chapel Hill, CB\#3250 Phillips Hall, Chapel Hill, NC 27599 